\title{
Anomalous Spin Response and Virtual-Carrier-Mediated Magnetism in a Topological Insulator
}

\author{
T. Kernreiter, ${ }^{1}$ M. Governale, ${ }^{1, *}$ U. Zülicke, ${ }^{1, \dagger}$ and E. M. Hankiewicz ${ }^{2}$ \\ ${ }^{1}$ School of Chemical and Physical Sciences and MacDiarmid Institute for Advanced Materials and \\ Nanotechnology, Victoria University of Wellington, P.O. Box 600, Wellington 6140, New Zealand \\ ${ }^{2}$ Institut für Theoretische Physik und Astrophysik (TP4), Universität Würzburg, \\ 97074 Würzburg, Germany
}

(Received 29 June 2015; published 18 April 2016)

\begin{abstract}
We present a comprehensive theoretical study of the static spin response in HgTe quantum wells, revealing distinctive behavior for the topologically nontrivial inverted structure. Most strikingly, the $\mathbf{q}=0$ (long-wavelength) spin susceptibility of the undoped topological-insulator system is constant and equal to the value found for the gapless Dirac-like structure, whereas the same quantity shows the typical decrease with increasing band gap in the normal-insulator regime. We discuss ramifications for the ordering of localized magnetic moments present in the quantum well, both in the insulating and electron-doped situations. The spin response of edge states is also considered, and we extract effective Landé $g$ factors for the bulk and edge electrons. The variety of counterintuitive spin-response properties revealed in our study arises from the system's versatility in accessing situations where the charge-carrier dynamics can be governed by ordinary Schrödinger-type physics; it mimics the behavior of chiral Dirac fermions or reflects the material's symmetry-protected topological order.
\end{abstract}

DOI: 10.1103/PhysRevX.6.021010

\section{INTRODUCTION AND MAIN RESULTS}

The two-dimensional (2D) topological insulator (TI) realized in inverted $\mathrm{HgTe}$ quantum wells exhibits unusual electric-transport properties [1-4] that have attracted great interest [5]. TI behavior is also found in other 2D [6,7] and bulk $[5,8]$ materials. The potential for interesting interplay between a TI's electronic and magnetic degrees of freedom, e.g., through hyperfine interaction with the material's nuclei [9], or local exchange interaction with magnetic dopants [10], has been pointed out recently $[11,12]$. These efforts have opened up new perspectives and extended previous work devoted to understanding spin effects $[13,14]$ and magnetism [15] in ordinary semiconductors. Our present theoretical study of the spin response in $\mathrm{HgTe}$ quantum wells reveals unconventional spin-related properties that distinguish this paradigmatic TI material from all other currently known 2D electronic systems. We thus provide alternative means for the experimental identification of the topological regime and extend current knowledge about the fundamentals of spin-response behavior in solids.

\footnotetext{
*michele.governale@vuw.ac.nz †uli.zuelicke@vuw.ac.nz
}

Published by the American Physical Society under the terms of the Creative Commons Attribution 3.0 License. Further distribution of this work must maintain attribution to the author(s) and the published article's title, journal citation, and DOI.
Subject Areas: Magnetism, Nanophysics,

Topological Insulators
The spin susceptibility contains comprehensive information about the magnetic properties of a material. In the simplest case of a spin-rotationally invariant noninteracting electron gas, the spin susceptibility is proportional to the charge-response (Lindhard) function [16]. Noticeable deviations from that situation occur, e.g., in systems with strong spin-orbit coupling such as 2D hole gases [17]. For metals or degenerately doped semiconductors, the spin response of only the partially filled band is typically considered. This approach misses intrinsic contributions to many-particle response functions arising from virtual interband transitions that become important in narrow-gap and, especially, gapless electron systems. Examples for the latter are the 2D Dirac-like electron states on the surface of a bulk TI whose magnetic properties have been discussed in Refs. $[18,19]$. The HgTe quantum wells considered here present an ideal testing ground for exploring the importance and properties of intrinsic, or virtual-carrier, effects, as it is possible to tune the band gap in such systems with a single structural parameter (i.e., the quantum-well width $d$ ). Furthermore, deviations from the conical 2D-Dirac dispersion in the gapless case are well characterized within a continuummodel (BHZ) description [20], which also gives controlled access to the full range of, and interesting transitions between, Schrödinger-physics-dominated, chiral-Diracfermion-like, and topologically nontrivial phases. In particular, definite (i.e., cutoff-independent) results for the intrinsic response at long wavelengths are obtained even in the limit of vanishing band gap-unlike in the case of the 
previously considered 2D-Dirac models used to describe the surface states of bulk TIs $[18,19]$.

Before going into greater detail in the remainder of this article, we briefly highlight four major advances and central new insights gained from our work.

(i) We find an analytical expression for the uniform static spin susceptibility of the intrinsic (undoped) system,

$$
\bar{\chi}_{x x, z z}^{(\mathrm{int})}(\gamma ; \mathbf{q}=0)=-\frac{\mathcal{C}_{x, z}^{2}(\gamma)}{16 \pi|B|} \frac{1}{1+4 \xi_{\mathrm{M}} \Theta\left(\xi_{\mathrm{M}}\right)},
$$

where $\xi_{\mathrm{M}}$ is proportional to the band gap and positive (negative) for the topologically trivial normal (topologically nontrivial inverted) regime, $\Theta$ denotes the Heaviside step function, $B$ is a bandstructure parameter from the BHZ model introduced below, and the constants $\mathcal{C}_{x, z}(\gamma)$ depend both on the valence-band mixing of quantum-well basis states and on the parameter $\gamma$ that characterizes the relative coupling strength of the conduction-band and valence-band spin degree of freedom to the physical quantity of interest. While the intrinsic spin response is suppressed with increasing band gap in the normal regime $\left(\xi_{M}>0\right)$, it becomes independent of gap size in the topological regime where $\xi_{M} \leq 0$. This unexpected behavior is a special feature of the symmetry-protected topological phase of the bulk system associated with the existence of gapless edge states.

(ii) A physical consequence of the unusual spin response in the intrinsic system is the asymmetric variation of the critical (Curie) temperature for virtual-carrier-mediated magnetic order in a $\mathrm{HgTe}$ quantum well that has been doped magnetically but not electronically. See Fig. 1(a). A similarly striking asymmetry arises when charge carriers are present in the 2D conduction band, which is illustrated in Fig. 1(b). Furthermore, in this situation, a rather strong, and counterintuitive, suppression of the Curie temperature with the density of itinerant charge carriers is revealed. This tendency arises from the unconventional character of conductionband states in the topological regime. See Sec. III B for details.

(iii) We extract the effective $g$ factors for 2D conduction electrons in both the topological and normal regimes, which are directly accessible experimentally [4]. Their strong density and gap-parameter dependencies reflect the band mixing and transition between regimes dominated by Schrödinger-type and Dirac-like dynamics of charge carriers. Full details can be found in Sec. IV. These results are essential, e.g., to enable quantitative characterization of the transition between quantum spin-Hall and quantum
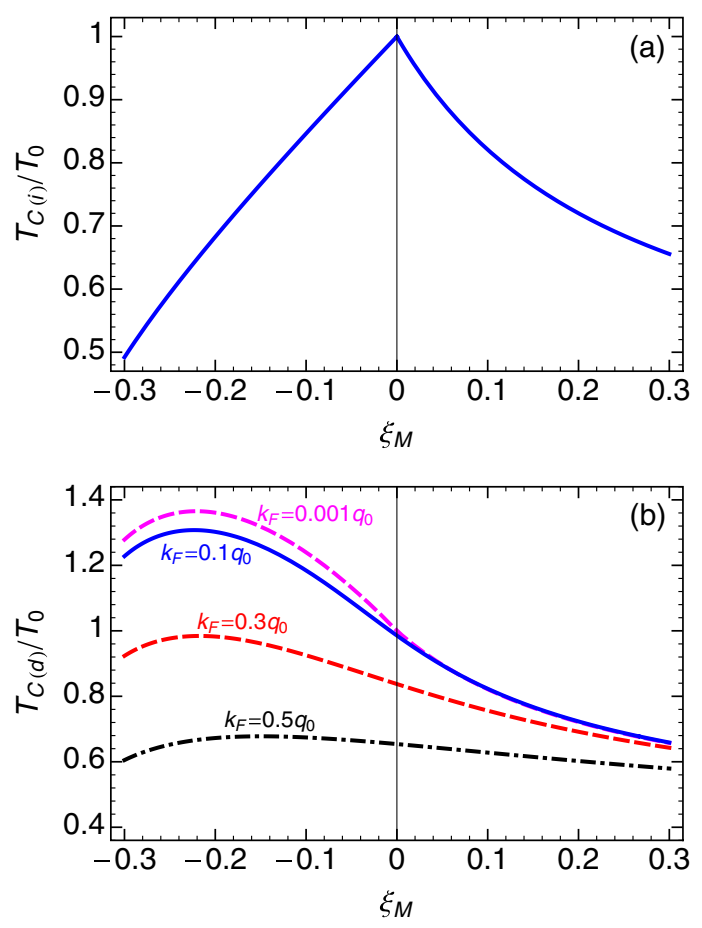

FIG. 1. (a) Mean-field critical temperature $T_{\mathrm{C}(\mathrm{i})}$ for virtualcarrier-mediated magnetism in a $\mathrm{HgTe}$ quantum well plotted as a function of the band-gap parameter $\xi_{\mathrm{M}}$. Note the striking asymmetry between the topological $\left(\xi_{M}<0\right)$ and normal $\left(\xi_{M}>0\right)$ regimes. An explicit expression for $T_{0}$ is given in the text; it contains the local exchange coupling between magnetic-impurity orbitals and HgTe quantum-well basis states, which is roughly constant across the transition [21]. (b) Critical temperature $T_{\mathrm{C}(\mathrm{d})}$ for the electron-doped $\mathrm{HgTe}$ quantum well plotted as a function of $\xi_{\mathrm{M}}$ and for various charge-carrier densities [corresponding to indicated values for the Fermi wave vector $k_{\mathrm{F}}$, where $k_{\mathrm{F}}=q_{0}$ corresponds to a charge density $n_{0} \equiv q_{0}^{2} /(2 \pi)=4.25 \times$ $10^{12} \mathrm{~cm}^{-2}$ for a typical structure [22]]. We used $\xi_{\mathrm{D}}=-0.7$ for the BHZ-model electron-hole-asymmetry parameter in both plots, and $\gamma=-2.22$ as relevant for the (Hg,Cd,Mn)Te system [21].

anomalous Hall phases in magnetic 2D topological insulators [11].

(iv) We predict the effective $g$ factors of the helical edge states that are present in the topological regime of the HgTe quantum well. For the out-of-plane magnetic-field direction, the $g$ factor assumes a constant value that is determined by band mixing in the quantum-well eigenstates. In contrast, the in-plane $g$ factor has a strong density dependence. See Sec. V.

In the following, we discuss relevant details of our theoretical analysis and present complete results for the spin response in both intrinsic and electron-doped systems.

\section{QUANTUM-WELL BAND STRUCTURE}

The electronic properties of $\mathrm{HgTe}$ quantum wells are adequately captured by an effective four-band (BHZ) Hamiltonian [20] that acts in the low-energy subspace 
spanned by basis states $\left|E_{1}+\right\rangle,\left|H_{1}+\right\rangle,\left|E_{1}-\right\rangle,\left|H_{1}-\right\rangle$ and explicitly reads

$$
\mathcal{H}_{0}=\left(\begin{array}{cc}
\mathcal{H}(\mathbf{k}) & 0 \\
0 & \mathcal{H}^{*}(-\mathbf{k})
\end{array}\right)
$$

with $\mathcal{H}(\mathbf{k})=h_{\mu} \sigma^{\mu}, h=\left(C-D k^{2}, A k_{x},-A k_{y}, M-B k^{2}\right)$ and $\sigma^{\mu}=(\mathbb{1}, \boldsymbol{\sigma})$. The parameters $A, B, C, D, M$ are functions of the well width $d$, and their numerical values are typically obtained by a fitting procedure [23]. The parameter $M$ opens a band gap, where (in the convention $B<0$ ) the system is in the topological (normal) regime when $M<0(M>0)$. The basis functions $\left|E_{1} \pm\right\rangle$ are a superposition of conductionelectron and light-hole (LH) basis functions with a given spin projection. The associated band $\left|L_{1} \pm\right\rangle$ has a much lower band-edge energy and can therefore be omitted. On the other hand, the heavy-hole $(\mathrm{HH})$ states $\left|H_{1} \pm\right\rangle$ also belong to the set of low-energy excitations. In the following, we set $C=0$ and employ a dimensionless description of Eq. (2) that is obtained by defining an energy scale $E_{0} \equiv A q_{0}$ and a scale for the wave vector $q_{0} \equiv A /|B|[24,25]$. (In a typical HgTe quantum-well structure [22], $E_{0}=0.189 \mathrm{eV}$ and $q_{0}=0.517 \mathrm{~nm}^{-1}$.) By making use of the axial symmetry of the BHZ Hamiltonian (2), we rotate to a real basis to obtain $\mathcal{H}^{\prime}(\mathbf{k})=U_{\phi_{\mathbf{k}}}^{(+) \dagger} \mathcal{H}(\mathbf{k}) U_{\phi_{\mathbf{k}}}^{(+)}=E_{0} h_{\mu}^{\prime} \sigma^{\mu}$, with $U_{\phi_{\mathbf{k}}}^{(s)}=$ $\operatorname{diag}\left(\mathrm{e}^{i s \phi_{\mathbf{k}} / 2}, \mathrm{e}^{-i s \phi_{\mathbf{k}} / 2}\right) \quad$ and $\quad h^{\prime}=\left(-\xi_{\mathrm{D}} \tilde{k}^{2}, \tilde{k}, 0, \xi_{\mathrm{M}}+\tilde{k}^{2}\right)$, where $\phi_{\mathbf{k}}$ is the polar angle of the $2 \mathrm{D}$ wave vector $\mathbf{k}$, and $\tilde{k}=k / q_{0}$. We have defined the dimensionless parameters $\xi_{\mathrm{M}} \equiv M / E_{0}$ and $\xi_{\mathrm{D}} \equiv D /|B|$, which have typical values $[20,22,23]\left|\xi_{\mathrm{M}}\right|,\left|\xi_{\mathrm{D}}\right| \lesssim 0.5$. The eigenvectors in the complex and real bases are related via $a_{\mathbf{k} \alpha}^{(s)}=U_{\phi_{\mathbf{k}}}^{(s)} a_{k \alpha}^{(s)}$, where $\alpha= \pm$ distinguishes the conduction and valence bands, which are doubly degenerate in the quantum number $s= \pm$ for spin projection along the growth direction and have the dispersions

$$
E_{\mathbf{k} \alpha}^{(s)} \equiv E_{k \alpha}^{(s)}=E_{0}\left[-\tilde{k}^{2} \xi_{\mathrm{D}}+\alpha \sqrt{\left(\xi_{\mathrm{M}}+\tilde{k}^{2}\right)^{2}+\tilde{k}^{2}}\right] .
$$

\section{EFFECTIVE SPIN SUSCEPTIBILITY OF THE 2D SYSTEM: INTRINSIC AND DOPED CASES}

The spin susceptibility is used to characterize a system's response to spin-related external stimuli within the framework of linear-response theory [16]. In the static limit, and for quantum-well-confined electrons, it can be written as [17]

$$
\begin{aligned}
& \chi_{i j}\left(\mathbf{R}, z ; \mathbf{R}^{\prime}, z^{\prime}\right) \\
& \quad=\lim _{\eta \rightarrow 0^{+}}\left\{-\frac{i}{\hbar} \int_{0}^{\infty} d t \mathrm{e}^{-\eta t}\left\langle\left[S_{i}(\mathbf{R}, z ; t), S_{j}\left(\mathbf{R}^{\prime}, z^{\prime} ; 0\right)\right]\right\rangle\right\},
\end{aligned}
$$

with $\mathbf{R} \equiv(x, y)$ and $z$ being coordinates in the 2D plane and perpendicular to it, respectively, and $S_{j}(\mathbf{R}, z)=$ $\Psi^{\dagger}(\mathbf{R}, z) \hat{S}_{j} \Psi(\mathbf{R}, z)$ denoting the electron spin density measured in units of $\hbar$. For a homogeneous 2D electron system, the spin susceptibility is most straightforwardly obtained in terms of the spatially Fourier-transformed quantity $\chi_{i j}\left(\mathbf{q} ; z, z^{\prime}\right)$ via

$$
\chi_{i j}\left(\mathbf{R}, z ; \mathbf{R}^{\prime}, z^{\prime}\right)=\int \frac{d^{2} q}{(2 \pi)^{2}} \mathrm{e}^{i \mathbf{q} \cdot\left(\mathbf{R}-\mathbf{R}^{\prime}\right)} \chi_{i j}\left(\mathbf{q} ; z, z^{\prime}\right) .
$$

The dependence of $\chi_{i j}\left(\mathbf{q} ; z, z^{\prime}\right)$ on the coordinates $z$ and $z^{\prime}$ encodes the spatial profile of the quantum-well bound states. Within the BHZ framework, the $z$-dependent part of electron wave functions is contained in the four basis functions $\left|E_{1} \pm\right\rangle,\left|H_{1} \pm\right\rangle$. The latter are spinors whose explicit form has been derived [20] within the six-band Kane-model description [26] for the charge-carrier dynamics that includes the bands with $\Gamma_{6}$ and $\Gamma_{8}$ symmetry closest to the bulk material's fundamental gap. As is generally the case in multiband systems, the spin response of electrons in a HgTe quantum well is strongly influenced by both the in-plane dynamics described by the BHZ Hamiltonian and the nontrivial spinor structure of the BHZ-model basis states. We therefore need to express the spin susceptibility within the underlying six-band Kane model.

The coupling between some physical stimulus represented by a field $\mathcal{F}$ and the $\Gamma_{6}$ and $\Gamma_{8}$-band intrinsic angular-momentum degrees of freedom $\hat{\boldsymbol{\sigma}}$ and $\hat{\mathbf{J}}$ is most generally described by a term

$$
\mathcal{H}_{\mathcal{F}}=\sum_{i} \mathcal{F}_{i}\left(b_{\Gamma_{6}} \frac{\hat{\sigma}_{i}}{2} \oplus 0_{4 \times 4}+b_{\Gamma_{8}} 0_{2 \times 2} \oplus \hat{J}_{i}\right)
$$

in the Kane-model Hamiltonian. See, e.g., Table C.5 in Ref. [26]. Within this approach, the coefficients $b_{\Gamma_{j}}$ are intraband coupling constants with appropriately renormalized values to take into account all field-induced bandcoupling effects in the bulk material. To be able to discuss a wide range of spin-related phenomena, we define an effective (pseudo)spin operator

$$
\hat{S}_{i}(\gamma)=\frac{\hat{\sigma}_{i}}{2} \oplus\left(\gamma \hat{J}_{i}\right),
$$

such that $\mathcal{H}_{\mathcal{F}} \equiv b_{\Gamma_{6}} \sum_{i} \mathcal{F}_{i} \hat{S}_{i}\left(b_{\Gamma_{8}} / b_{\Gamma_{6}}\right)$. The actual value of the parameter $\gamma$ depends on the physical quantity or situation of interest [27]. The system's response is then fully captured by the effective spin-susceptibility tensor

$$
\begin{aligned}
\chi_{i j}\left(\gamma ; \mathbf{q} ; z, z^{\prime}\right)= & \sum_{\alpha, \beta, s, s^{\prime}} \int \frac{d^{2} k}{(2 \pi)^{2}} \mathcal{W}_{i j(\mathbf{k}, \mathbf{k}+\mathbf{q}, \alpha, \beta)}^{\left(s, s^{\prime}\right)}\left(\gamma ; z, z^{\prime}\right) \\
& \times \frac{n_{\mathrm{F}}\left(E_{\mathbf{k} \alpha}^{(s)}\right)-n_{\mathrm{F}}\left(E_{\mathbf{k}+\mathbf{q} \beta}^{\left(s^{\prime}\right)}\right)}{E_{\mathbf{k} \alpha}^{(s)}-E_{\mathbf{k}+\mathbf{q} \beta}^{\left(s^{\prime}\right)}+i \hbar \eta},
\end{aligned}
$$


where $n_{\mathrm{F}}$ denotes the Fermi function, and

$$
\begin{aligned}
\mathcal{W}_{i j(\mathbf{k}, \mathbf{k}+\mathbf{q}, \alpha, \beta)}^{\left(s, s^{\prime}\right)}\left(\gamma ; z, z^{\prime}\right)= & {\left[\psi_{\mathbf{k} \alpha}^{(s)}(z)\right]^{\dagger} \cdot\left[\hat{S}_{i}(\gamma) \psi_{\mathbf{k}+\mathbf{q} \beta}^{\left(s^{\prime}\right)}(z)\right] } \\
& \times\left[\psi_{\mathbf{k}+\mathbf{q} \beta}^{\left(s^{\prime}\right)}\left(z^{\prime}\right)\right]^{\dagger} \cdot\left[\hat{S}_{j}(\gamma) \psi_{\mathbf{k} \alpha}^{(s)}\left(z^{\prime}\right)\right]
\end{aligned}
$$

are matrix elements of the effective spin operator given in Eq. (7). In the spirit of subband $\mathbf{k} \cdot \mathbf{p}$ theory, the sixdimensional spinor wave functions $\psi_{\mathbf{k} \alpha}^{(s)}(z)$ can be expressed in terms of the BHZ-model basis state spinors $\psi_{0 i}^{(s)}(z)$ as

$$
\psi_{\mathbf{k} \alpha}^{(s)}(z)=\sum_{i=1}^{2}\left(U_{\phi_{\mathbf{k}}}^{(s)}\right)_{i i} a_{k \alpha, i}^{(s)} \psi_{0 i}^{(s)}(z),
$$

where the coefficients $a_{k \alpha, i}^{(s)}$ are the components of the corresponding eigenvectors of the BHZ Hamiltonian (see Sec. II). The explicit form of the basis states was derived in Ref. [20] by solving a confined-particle problem in the $\mathrm{HgTe} / \mathrm{CdTe}$ hybrid system. For instance, $\psi_{01}^{(+)}(z)^{T}=$ $\left(f_{1}(z), 0,0, f_{4}(z), 0,0\right)$, with the detailed form of the envelope function components $f_{i}(z)$ provided in the supplemental information of Ref. [20].

In the following, we consider the growth-directionaveraged spin susceptibility of charge carriers in the HgTe quantum well, given by $\bar{\chi}_{i j}(\gamma ; \mathbf{q})=\int d z \int d z^{\prime} \chi_{i j}$ $\left(\gamma ; \mathbf{q} ; z, z^{\prime}\right)$ [17], with $\chi_{i j}\left(\gamma ; \mathbf{q} ; z, z^{\prime}\right)$ calculated using the Kane-model-based BHZ approach as described above. Note that, by replacing the spin matrices in Eq. (8b) by the unit matrix and averaging over $z, z^{\prime}$, we obtain the charge-response function studied in Refs. [24,25]. From the axial symmetry of our Kane-model description, and the fact that the eigenstates have definite spin projection in the growth direction, it follows that the in-plane spin susceptibilities are the same, i.e., $\bar{\chi}_{x x}(\gamma ; q)=\bar{\chi}_{y y}(\gamma ; q)$, and they only depend on the magnitude $q \equiv|\mathbf{q}|$. This is an important difference from other semiconducting systems, where HH-LH mixing occurs [17]. In the present situation, HH-LH mixing arises only from terms linear in $\mathbf{k}$ (giving rise to Dirac-like excitations), which is a consequence of the envelope function components [20] behaving differently (even or odd) under the parity transformation $z \leftrightarrow-z$.

\section{A. Spin response of the intrinsic system}

In the insulating limit, the conduction band is empty and the Fermi level lies in the band gap, i.e., $|\mu|<E_{0}\left|\xi_{\mathrm{M}}\right|$. In this situation, the spin susceptibility originates from virtual interband transitions across the band gap. In the following, we focus on the zero temperature limit. The analytical result for the intrinsic spin susceptibility obtained in the limit of zero momentum transfer $q \rightarrow 0$ is given in Eq. (1), where

$$
\begin{gathered}
\mathcal{C}_{x}(\gamma)=1+(2 \gamma-1) \mathcal{C}_{\mathrm{LH}}, \\
\mathcal{C}_{z}(\gamma)=1-3 \gamma+(\gamma-1) \mathcal{C}_{\mathrm{LH}},
\end{gathered}
$$

and $\mathcal{C}_{\mathrm{LH}} \equiv \int d z\left|f_{4}(z)\right|^{2} \quad(\approx 0.4)$ is the amount of $\mathrm{LH}$ admixture in the basis states $\left|E_{1} \pm\right\rangle$. (Details about the calculation of the intrinsic spin susceptibility are given in Appendix A. Results for $q \neq 0$ can be calculated numerically. For completeness, some of these are also shown in Appendix A.) Equation (1) exhibits anomalous behavior in the inverted region $\left(\xi_{\mathrm{M}}<0\right)$ in that the uniform spin response is independent of the gap size and pinned to the value for the gapless case, even though it arises from virtual interband transitions. In the normal region $\left(\xi_{\mathrm{M}}>0\right)$, the expected decrease of the response functions with increasing band gap is found. The spin susceptibility (1) is strongly anisotropic, generally exhibiting a dominant out-of-plane response except for a very small range of the parameter $\gamma$. See Fig. 2.

A nonvanishing $\bar{\chi}_{j j}^{(\mathrm{int})}(\gamma ; 0)$ seems to imply the counterintuitive phenomenon that an applied magnetic field could generate a magnetization of the HgTe quantum-well system in the intrinsic limit where no charge carriers are present. Our more detailed analysis shows, however, that this is not the case. Direct calculation of the derivative of the free energy with respect to magnetic-field strength in a model based on the BHZ Hamiltonian (2) augmented by a Zeeman term reveals that the total magnetization is strictly zero [28]. This is due to the fact that intrinsic-spin and orbital contributions to the magnetization cancel, as expected in a spin-orbit-coupled system [29]. This conclusion is further underpinned by the observation that $\bar{\chi}_{j j}^{\text {(int) }}(\gamma ; 0)$ vanishes in the limit of zero HH-LH mixing [30].

As it is possible to engineer and study effective exchange interactions between impurity atoms [31,32], it is tempting to consider such interactions between two localized spins in a HgTe quantum well. Quite generally, the RKKY Hamiltonian is given by [33]

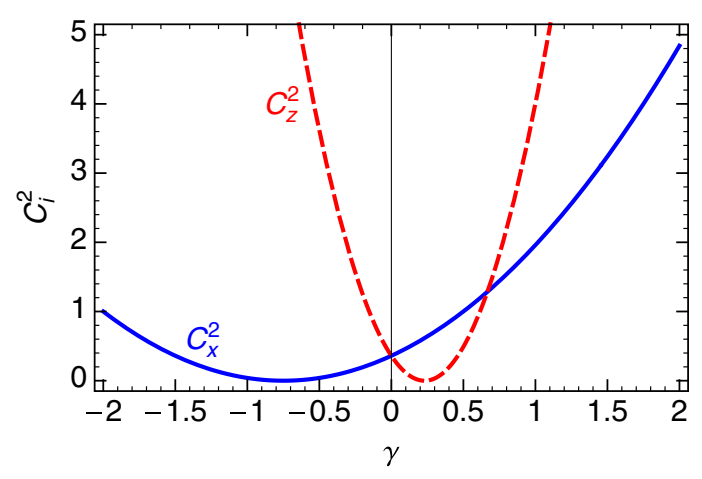

FIG. 2. Prefactors $C_{x}^{2}$ and $C_{y}^{2}$ determining the magnitude of intrinsic in-plane and out-of-plane spin response, respectively [see Eq. (1)], plotted as a function of the parameter $\gamma$ from the definition of the effective Kane-model spin operator (7). 


$$
\mathcal{H}_{\mathbf{r}, \mathbf{r}^{\prime}}^{\text {eff }}=G^{2} \sum_{i, j} I_{\mathbf{r}}^{i} I_{\mathbf{r}^{\prime}}^{j} \chi_{i j}\left(\mathbf{r}, \mathbf{r}^{\prime}\right)
$$

Here, $\chi_{i j}\left(\mathbf{r}, \mathbf{r}^{\prime}\right)$, the Fourier transform of Eq. (8a), is the spin susceptibility in real space, $I_{\mathbf{r}}^{i}$ denotes the $i$ th Cartesian component of an impurity spin located at position $\mathbf{r}=(\mathbf{R}, z)$, and $G$ is the local exchange-coupling constant between the spin degree of freedom carried by band electrons and localized (e.g., impurity) spins. (The difference in exchange-coupling strengths for conduction-band and valence-band states is accounted for by the appropriate value of $\gamma$.) In Fig. 3(a), we plot the growth-directionaveraged effective out-of-plane real-space spin susceptibility $\bar{\chi}_{z z}(R)$ as a function of the distance $R$ for various values of $\xi_{\mathrm{M}}$ (and $\xi_{\mathrm{D}}=-0.7$ corresponding to realistic situations [23]).

Figure 3 shows that the effective exchange interaction mediated by the intrinsic spin response of the $\mathrm{HgTe}$ quantum well for localized spins is of ferromagnetic (FM) type if $R q_{0} \ll 1$, and that there is a crossover to antiferromagnetic (AFM) coupling for $R q_{0} \gtrsim 1$ that sensitively depends on $\xi_{\mathrm{M}}$. In particular, we find that this crossover is shifted significantly to smaller impurity-spin separations in the case of the inverted regime as compared to the normal regime. Also, the magnitude of exchange interaction in the ferromagnetic regime for fixed distance $R$
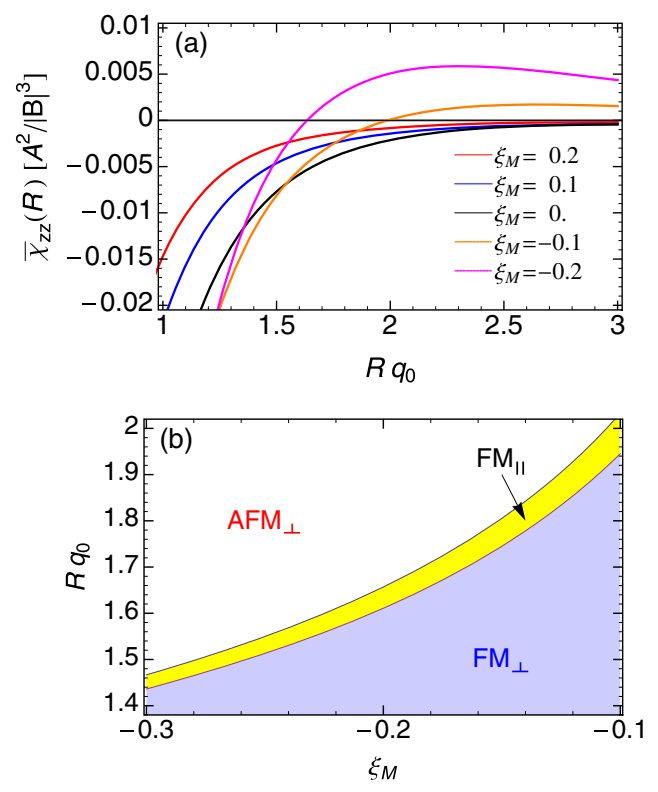

FIG. 3. (a) Intrinsic real-space spin susceptibility $\bar{\chi}_{z z}(R)$ plotted as a function of $R q_{0}$ for several values of the gap parameter $\xi_{\mathrm{M}}$, with fixed $\xi_{\mathrm{D}}=-0.7$ and $\gamma=-2.22$. (b) Parameter domains of preferential alignments between localized Mn spins having a distance $R$, mediated by the intrinsic spin response of an $\mathrm{HgTe}$ quantum-well system. The blue (white) domain favors a ferromagnetic (antiferromagnetic) out-of-plane spin alignment. In the yellow region, the two localized spins tend to align ferromagnetically in-plane. markedly increases when enlarging the gap in the inverted system. In contrast, the exchange interactions in the normal regime have a very small magnitude. To further illustrate the parametric dependencies of the carrier-mediated exchange interaction, Fig. 3(b) shows the various regions of preferential spin alignments as a function of $\xi_{\mathrm{M}}<0$. Interestingly, in between the two out-of-plane localizedspin alignments $\left(\mathrm{FM}_{\perp}\right.$ and $\left.\mathrm{AFM}_{\perp}\right)$, we also find sizable regions for the distance where it is energetically favorable for impurity spins to align ferromagnetically in-plane $\left(\mathrm{FM}_{\|}\right)$. Moreover, for larger distances, we find the usual Bloembergen-Rowland [34] behavior for the intrinsic spin susceptibilities, which is characterized by an exponential decay factor that depends on the band gap, approximately given by $\sim \exp \left(-R / R_{0}\right) / R^{3}$, where $R_{0}=\left(\left|\xi_{\mathrm{M}}\right| q_{0}\right)^{-1}$ is the Compton wavelength of the band electrons. More details are given in Appendix B. In the limit of zero gap $\left(\xi_{\mathrm{M}} \rightarrow 0\right)$, the $R^{-3}$ decay found previously in various Dirac systems $[18,19,35]$ is reproduced.

Having considered the case of two localized impurity spins with exchange interactions mediated by virtual excitations, we now focus on a HgTe quantum well that is doped with a large number of homogeneously distributed magnetic impurities (e.g., Mn ions), forming effectively a $\mathrm{Hg}_{1-x} \mathrm{Mn}_{x}$ Te alloy [36]. The average distance between the spins is $R_{\mathrm{NN}} \approx\left(3 a_{0}^{3} / 16 \pi x\right)^{1 / 3}$ [37], with $a_{0}$ the $\mathrm{HgTe}$ lattice constant and $x$ the concentration of magnetic ions. We calculate the Curie temperature in the mean-field limit, assuming $R_{\mathrm{NN}} / R_{0} \ll 1$, which is justified when $x \gg 2 \times 10^{-3}\left|\xi_{\mathrm{M}}\right|^{3}$, where we have used $q_{0} \lesssim 0.5 \mathrm{~nm}^{-1}$. Since $\left|\xi_{\mathrm{M}}\right| \lesssim 0.3$ [23], this condition is practically always fulfilled. The Curie temperature for Ising-type ferromagnetic order with magnetization perpendicular to the quantum-well growth direction is given by

$$
T_{\mathrm{C}(\mathrm{i})}=T_{0} \frac{d_{\mathrm{c}} / d\left(\xi_{\mathrm{M}}\right)}{1+4 \xi_{\mathrm{M}} \Theta\left(\xi_{\mathrm{M}}\right)},
$$

where $T_{0}=\left\{\left[I(I+1) \mathcal{C}_{z}^{2}(\gamma)\right] /(48 \pi)\right\}\left[G^{2} /\left(k_{\mathrm{B}}|B|\right)\right]\left(n_{I} / d_{\mathrm{c}}\right)$, $I$ denotes the impurity-spin magnitude, $n_{I}$ is the $3 \mathrm{D}$ density of magnetic impurities, and $d_{\mathrm{c}} \approx 6.3 \mathrm{~nm}$ is the critical well width. For obtaining Eq. (12), we have used the approximation $\int_{-d / 2}^{d / 2} d z \int_{-d / 2}^{d / 2} d z^{\prime} \chi_{j j}^{(\text {int })}\left(\gamma ; 0, z, z^{\prime}\right) \approx \bar{\chi}_{j j}^{\text {(int) }}(\gamma ; 0)$. In Fig. 1(a), we show the Curie temperature as a function of $\xi_{\mathrm{M}}$, where we set $A=0.375 \mathrm{eVnm}$ and $B=$ $-1.120 \mathrm{eV} \mathrm{nm}^{2}$ [23] because the variation of $A$ and $B$ with $d$ is much weaker than that of $M$. We see that the behavior of the Curie temperature in its dependence on $\xi_{\mathrm{M}}$, or equivalently $d$, provides a clear means to distinguish between topological and normal regions.

Knowing the spin susceptibility as a function of the wave vector allows us to go beyond the mean-field limit $[13,14]$ and discuss the stability of the mean-field ground state with respect to thermally excited spin waves (magnons). In our 
present case of interest, the magnon dispersion for $q / q_{0} \ll 1$ is given by $\omega_{q}=\omega_{0}+c_{2} q^{2}$, with a coefficient $c_{2}=\left.\frac{1}{2}\left\{\left[\partial^{2} \bar{\chi}_{z z}^{(\text {int })}(\gamma ; q)\right] /\left(\partial q^{2}\right)\right\}\right|_{q=0}$. Using Fig. 6 in Appendix A, which shows results for the relevant set of parameters, we find $c_{2} \lesssim 0$. Thus, the criterion [14,17,33] $c_{2}>0$ needed to guarantee stability of the mean-field ground state is generally violated. It may be possible that electron-electron interactions and/or spin-orbit coupling help to stabilize ferromagnetic order, as has been shown to be the case for an ordinary 2D electron gas $[13,14,38]$, but this question is beyond the scope of our present work.

\section{B. Spin response of the electron-doped system}

We now consider situations where the conduction band is occupied $\left(\mu>E_{0}\left|\xi_{\mathrm{M}}\right|\right)$. In the $q \rightarrow 0$ limit, an analytical expression for the extrinsic contribution to the spin susceptibility (arising from filled conduction-band states) is found as

$$
\begin{aligned}
\bar{\chi}_{x x}^{(\mathrm{dop})}(\gamma ; 0)= & \frac{\mathcal{C}_{x}^{2}(\gamma)}{16 \pi|B|}\left[\frac{2 \pi \tilde{N}(0)}{f^{2}}\left(\tilde{k}_{\mathrm{F}}^{2}-2 f\left(\tilde{k}_{\mathrm{F}}^{2}+\xi_{\mathrm{M}}\right)-2 f^{2}\right)\right. \\
& \left.+\frac{\left.\tilde{k}_{\mathrm{F}}^{2}\left(1+2 \xi_{\mathrm{M}}\right)+2 \xi_{\mathrm{M}}^{2}-2 f\left|\xi_{\mathrm{M}}\right|\right)}{f\left(1+4 \xi_{\mathrm{M}}\right)}\right], \quad(13 \mathrm{a}) \\
\bar{\chi}_{z z}^{(\mathrm{dop})}(\gamma ; 0)= & \frac{\mathcal{C}_{z}^{2}(\gamma)}{16 \pi|B|}\left[\frac { 2 \pi \tilde { N } ( 0 ) } { f ^ { 2 } } \left(\frac{6 \gamma \mathcal{D}_{z}(\gamma)}{\mathcal{C}_{z}^{2}(\gamma)} \tilde{k}_{\mathrm{F}}^{2}\right.\right. \\
& \left.-\frac{4 f^{2}\left\{\mathcal{D}_{z}^{2}(\gamma)\left(\tilde{k}_{\mathrm{F}}^{2}+\xi_{\mathrm{M}}+f\right)^{4}+9 \gamma^{2} \tilde{k}_{\mathrm{F}}^{4}\right\}}{\mathcal{C}_{z}^{2}(\gamma)\left[\tilde{k}_{\mathrm{F}}^{2}+\left(\tilde{k}_{\mathrm{F}}^{2}+\xi_{\mathrm{M}}+f\right)^{2}\right]^{2}}\right) \\
& \left.+\frac{\left.\tilde{k}_{\mathrm{F}}^{2}\left(1+2 \xi_{\mathrm{M}}\right)+2 \xi_{\mathrm{M}}^{2}-2 f\left|\xi_{\mathrm{M}}\right|\right)}{f\left(1+4 \xi_{\mathrm{M}}\right)}\right],
\end{aligned}
$$

where the first (second) term between the square brackets in the expression for $\bar{\chi}_{j j}^{\text {(dop) }}(\gamma ; 0)$ is an intraband (interband) contribution. In Eqs. (13), we used the abbreviations $\mathcal{D}_{z}(\gamma) \equiv(1-\gamma) \mathcal{C}_{\mathrm{LH}}-1$ and $f \equiv \sqrt{\left(\tilde{k}_{\mathrm{F}}^{2}+\xi_{\mathrm{M}}\right)^{2}+\tilde{k}_{\mathrm{F}}^{2}} . \tilde{N}(0)$ is related to the density of states at the Fermi energy as $-\bar{\chi}_{0}(0)=N(0)=(2 /|B|) \tilde{N}(0)$ [where $\bar{\chi}_{0}(q)$ is the static charge-response function], and it reads $\tilde{N}(0)=$ $\frac{1}{2 \pi}\left|\left[\left(1+2 \tilde{k}_{\mathrm{F}}^{2}+2 \xi_{\mathrm{M}}\right) /\left(\sqrt{\left(\tilde{k}_{\mathrm{F}}^{2}+\xi_{\mathrm{M}}\right)^{2}+\tilde{k}_{\mathrm{F}}^{2}}\right)\right]-2 \xi_{\mathrm{D}}\right|^{-1}$. For the general case with $q>0, \bar{\chi}_{j j}(\gamma ; q)$ can be calculated numerically, and its line shape is presented in Appendix C for various examples. Because of the sharpness of the Fermi surface, we find, for the corresponding real-space spin susceptibilities, the $R^{-2}$ oscillatory decay expected from a 2D Fermi liquid [16].

Next, we calculate the Curie temperature using the mean-field approach under the premise $k_{\mathrm{F}} R_{\mathrm{NN}} \ll 1$, which leads to the condition $x \gg 2 \times 10^{-3} \tilde{k}_{\mathrm{F}}$. This condition is generally fulfilled since $k_{\mathrm{F}} \lesssim 0.1 \mathrm{~nm}^{-1}$ is the reliable range of the effective model [23]. The Curie temperature is given by

$$
T_{\mathrm{C}(\mathrm{d})}=T_{0} \frac{d_{\mathrm{c}}}{d\left(\xi_{\mathrm{M}}\right)} \frac{16 \pi|B|}{C_{z}^{2}(\gamma)}\left|\bar{\chi}_{z z}(\gamma ; 0)\right|,
$$

where $\bar{\chi}_{z z}(\gamma ; 0)=\bar{\chi}_{z z}^{(\text {int })}(\gamma ; 0)+\bar{\chi}_{z z}^{(\text {dop })}(\gamma ; 0)$. Note that, also in the doped case, Ising-type magnetism prevails. In Fig. 1(b), we show the Curie temperature as a function of $\xi_{\mathrm{M}}$ for various values of the Fermi wave vector. For low doping, we see a strong dependence of the Curie temperature on $\xi_{\mathrm{M}}$ in the topological regime, which becomes very weak in the normal regime. For large doping, on the other hand, the Curie temperature is not very sensitive to $\xi_{\mathrm{M}}$ in its whole range. Furthermore, the Curie temperature is generally suppressed with increased doping, but this trend is much stronger in the topological regime. For the doped case, we find the magnon dispersion $\omega_{q}=$ $\omega_{0}+\bar{c}_{2} q^{2}$, where $\bar{c}_{2} \equiv \frac{1}{2}\left\{\left.\left[\left(\partial^{2} \bar{\chi}_{z z}(\gamma ; q)\right] /\left(\partial q^{2}\right)\right\}\right|_{q=0}>0\right.$ always, in contrast to the intrinsic case. See Fig. 8 in Appendix C. Thus, a necessary condition for the stability of the ferromagnetic ground state is fulfilled $[14,17,33]$.

\section{CONFINEMENT DEPENDENCE OF THE 2D-ELECTRON EFFECTIVE $g$ FACTOR}

The paramagnetic response of charge carriers in quantum-confined structures is usually interpreted in terms of the Pauli spin susceptibility and quantified by an effective single-particle $g$ factor [39-41]. However, any actually measured spin-related quantities almost always correspond to averaged collective responses of the, e.g., quasi-2D electron system that can be crucially affected by nontrivial spin-related phenomena [17]. Here, we consider the paramagnetic response of conduction-band electrons in HgTe quantum wells and show how their paramagnetic response is changed as a function of the band-gap parameter that drives the transition between the topological and normal regimes.

To define an effective $g$ factor for our system of a $\mathrm{HgTe}$ quantum well, we introduce the bulk-material Zeeman term $\mathcal{H}_{\mathcal{B}}=g_{*} \mu_{\mathrm{B}} \sum_{j} \mathcal{B}_{j} \hat{S}_{j}\left(-2 \kappa / g_{*}\right)$, where $\mu_{\mathrm{B}}$ is the Bohr magneton and $g_{*}(\kappa)$ the $\Gamma_{6}$-band ( $\Gamma_{8}$-band) $g$ factor [26]. Linear-response theory enables us to determine the paramagnetic response to the magnetic field, which is given by $\bar{\chi}_{\mathrm{P}, j}=\left.\left(g_{*} \mu_{\mathrm{B}}\right)^{2} \bar{\chi}_{j j}^{(\mathrm{dop})}\left(-2 \kappa / g_{*} ; q\right)\right|_{q=0}$, where $\bar{\chi}_{j j}^{(\mathrm{dop})}$ $\left(-2 \kappa / g_{*} ; q\right)$ are the spin susceptibilities of the electrondoped system for the in-plane and out-of-plane response involving $\hat{S}_{j}\left(-2 \kappa / g_{*}\right)$ [see Eq. (13) for $\gamma=-2 \kappa / g_{*}$ ]. We compare this with the Pauli susceptibility given by $\bar{\chi}_{\mathrm{P}, j}=\left(g_{j} \mu_{\mathrm{B}}\right)^{2} \bar{\chi}_{0}(0) / 4$, with $\bar{\chi}_{0}(0)$ being (up to a minus sign) the density of states which is the zero- $q$ limit of the Lindhard function and $g_{j}$ is the Lande $g$ factor for the two 
directions. Thus, we can extract collective $g$ factors for the charge carriers as

$$
g_{j}=g_{*} \sqrt{\left.4 \frac{\bar{\chi}_{j j}^{\text {dop })}\left(-2 \kappa / g_{*} ; q\right)}{\bar{\chi}_{0}(q)}\right|_{q=0}}
$$

Our approach to determine $g$ factors via the spin susceptibility complements previous work [42] where an effective Zeeman term was derived for the BHZ Hamiltonian.

For a narrow-gap semiconductor, the values for $g_{*}$ and $\kappa$ are dominated by band-coupling contributions. Using generic expressions resulting from Kane-model descriptions [26], we find

$$
\frac{2 \kappa}{g_{*}}=-\frac{\xi_{M}+\xi_{\Delta}}{2 \xi_{\Delta}}
$$

with $\xi_{\Delta}=\Delta_{0}|B| / A^{2}$ in terms of the spin-orbit-splitting gap $\Delta_{0}$ between the $\Gamma_{8}$ and $\Gamma_{7}$ band edges in the $8 \times 8$ Kane model. As $\xi_{\Delta} \gg\left|\xi_{M}\right|$ for our situations of interest, we can set $2 \kappa / g_{*} \rightarrow-0.5$ in the following. Hence, in Fig. 4, we show the effective $g$ factors for in-plane and out-of-plane responses as a function of $\xi_{\mathrm{M}}$ for various levels of electron doping obtained for $2 \kappa / g_{*}=-0.5$.
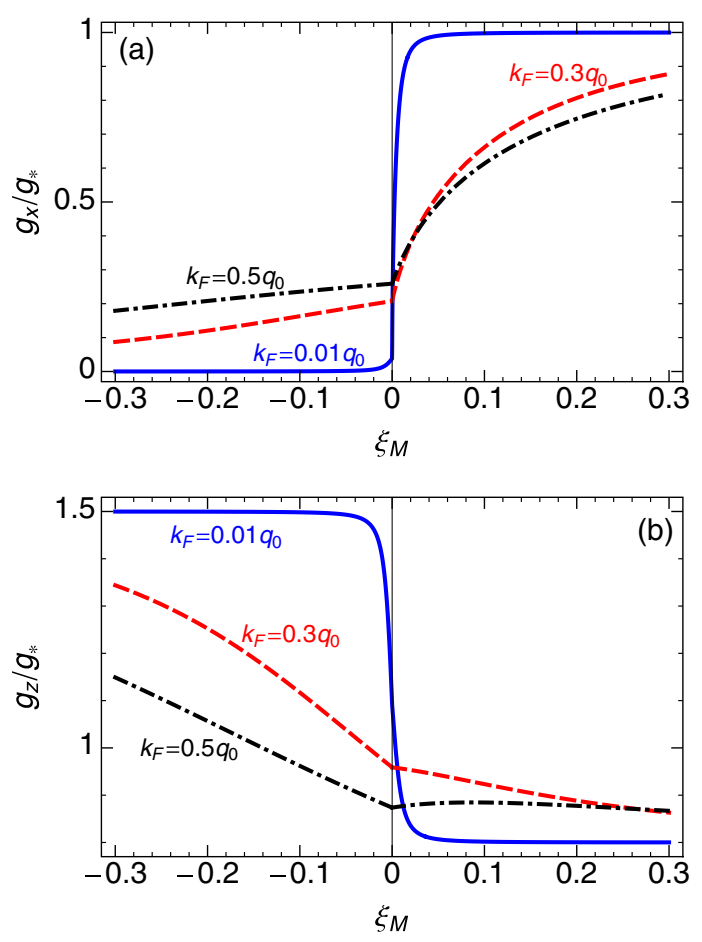

FIG. 4. Effective $g$ factors associated with (a) the in-plane and (b) the out-of-plane response of an electron-doped HgTe quantum well, plotted as a function of the gap parameter $\xi_{\mathrm{M}}$ for various levels of doping and with $\xi_{\mathrm{D}}=-0.7,2 \kappa / g_{*}=-0.5$. (Note that $k_{\mathrm{F}}=q_{0}$ corresponds to a charge density $n_{0} \equiv$ $q_{0}^{2} /(2 \pi)=4.25 \times 10^{12} \mathrm{~cm}^{-2}$ for a typical structure [22].)
For very low densities, we see a vanishing in-plane response and a maximal $g_{z}=6 \kappa$ HH-like out-of-plane response in the topological region $\xi_{\mathrm{M}}<0$. This behavior arises because the conduction-band character is dominated by the HH basis states, which experience a "frozen" spin orientation perpendicular to the quantum well due to the confinement-induced HH-LH energy splitting [43]. This can be easily verified from Eq. (13) by taking the limit $k_{\mathrm{F}} \rightarrow 0$. Departures from these results occur for larger doping levels (larger $k_{\mathrm{F}}$ ) due to increased HH-LH mixing. In the normal region $\left(\xi_{\mathrm{M}}>0\right)$, we encounter the situation in which the conduction band is dominantly composed of the electron basis states, rendering the $g$ factor sizable at any doping. The fact that the in-plane spin response is notably larger than the out-of-plane response for $\xi_{\mathrm{M}}>0$ and low doping is due to the $\mathrm{LH}$ admixture in the conduction-band states. As the carrier density increases, the concomitantly increased HH-LH mixing results in significant modifications. More detailed exploration of parametric dependencies exhibited by the collective $g$ factors obtained here will be useful to augment previous perturbative estimates [42] and aid the interpretation of recent measurements [4].t

\section{SPIN RESPONSE OF QUASI-1D HELICAL EDGE STATES IN THE TOPOLOGICAL REGIME}

We have also investigated the edge-state contributions to the spin susceptibility in the topological region, finding them to be negligible compared to the bulk contributions in almost all situations. The only exception occurs for the in-plane response function $\bar{\chi}_{x x}(\gamma ; q)$ in the purely intrinsic situation where the Fermi level lies in the minigap of the edge-state dispersions that opens up in a finite-size sample $[5,44]$. More details and full results pertaining to edge states are given in Appendixes D and E.

Up to very small finite-size corrections, the paramagnetic response of helical edge states is captured by using the effective $g$ factors $g_{x, z}^{(\mathrm{e})}$ given by

$$
\begin{gathered}
\frac{g_{x}^{(\mathrm{e})}}{g_{*}}=\sqrt{\frac{\mathcal{C}_{x}^{2}\left(-2 \kappa / g_{*}\right)}{4} \ln \left(\frac{\Lambda}{k_{\mathrm{F}}}\right) \stackrel{\frac{2 \kappa}{g_{*}}=-\frac{1}{2}}{\longrightarrow} \frac{\sqrt{\ln \left(\frac{\Lambda}{k_{\mathrm{F}}}\right)}}{2},} \\
\frac{g_{z}^{(\mathrm{e})}}{g_{*}}=-\frac{4 \kappa}{g_{*}}+\frac{1-\mathcal{C}_{\mathrm{LH}}}{2}\left(1+\frac{2 \kappa}{g_{*}}\right) \stackrel{\frac{2 \kappa}{g_{*}}=-\frac{1}{2}}{\longrightarrow} 1+\frac{1-\mathcal{C}_{\mathrm{LH}}}{4} .
\end{gathered}
$$

The results shown in Eqs. (17) were obtained using Eq. (15) together with Eqs. (E7a) and (E7b). The prediction of the edge-state $g$ factor is a major result of our work. In Fig. 5, we plot the in-plane $g$ factor as a function of $k_{\mathrm{F}}$, where we used the natural cutoff scale $\Lambda=\pi / a_{0}$. Interestingly, the in-plane $g$ factor of edge states decreases monotonically with increasing doping level. Such a behavior is in stark 


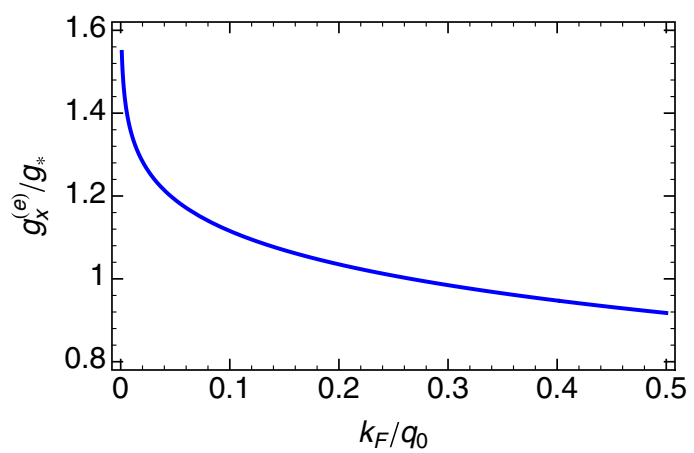

FIG. 5. Effective $g$ factor characterizing the response of helical edge states to an in-plane magnetic field.

contrast to the bulk case shown in Fig. 4(a) and to other 2D systems $[17,45]$ where the in-plane response increases with increased doping. This behavior reflects the fact that the spin-quantization axis of the edge states is perpendicular to the 2D plane, i.e., parallel to the $z$ direction, and their helical nature.

\section{CONCLUSIONS}

We present a detailed theoretical study of the spin response in HgTe quantum wells where virtual-carrierrelated processes are particularly relevant and exactly tractable within the effective BHZ-model description. Anomalous properties of the spin susceptibility and carrier-mediated magnetism are found in the inverted regime, extending our current understanding of spin-related properties in topological materials.

Most strikingly, the uniform static spin susceptibility in the intrinsic limit is constant, independent of the band gap, in the topological regime [see Eq. (1)]. This results in a distinct asymmetry between normal and inverted systems illustrated, e.g., by the dependence of the critical temperature for virtual-carrier-mediated magnetic order in a system that is doped with magnetic ions. Important differences between the two regimes are also exhibited in the situation where the conduction band becomes filled (see Fig. 1). The stability of mean-field ferromagnetic ground states with respect to thermal excitations of magnons has been analyzed. We find that the magnetic order is stable in the situation with finite doping.

In the topological regime, quasi-1D helical edge states exist. We have investigated their spin-response properties, finding that their contribution to the spin susceptibility is thermodynamically suppressed compared to that arising from 2D quantum-well states, except in the very specialand probably physically hard-to-realize-situation when the chemical potential is in the minigap opened by the hybridization of states from opposite edges in a finite sample.

We have used our results obtained for the spin susceptibility to define effective collective $g$ factors for states from the quasi-2D quantum-well subbands and also for the quasi-1D helical edge states. The different character of
quasi-2D-subband states in the normal and topological (inverted) regimes is reflected in the values for the effective $g$ factor. Their strong dependence on charge-carrier density reveals the importance of interband mixing. The behavior of the $g$ factors found for the edge states reflects their helical nature and spin-quantization property.

Our results are directly relevant for current and potential future experimental investigations of the spin-related properties of 2D topological insulators, in particular, those realized in $\mathrm{HgTe} / \mathrm{HgCdTe}$ and $\mathrm{InAs} / \mathrm{GaSb}$ quantum wells. For example, recent observation of Josephsonjunction interference patterns in an $\mathrm{S}-\mathrm{HgTe} / \mathrm{HgCdTe}-\mathrm{S}$ hybrid system has enabled extraction of $g$ factors for the quantum-well charge carriers [4]. It would be interesting to use similar techniques [46,47] to measure the edge-state $g$ factors and compare with our predictions. Furthermore, our results for the spin response in both the intrinsic and doped regimes are informative for the design of, and interpretation of measured quantities for, dilute magnetic phases in these systems $[10,15]$.

It would be interesting to extend our formalism to study the spin response in 3D topological-insulator materials [8]. In particular, as was the case in the 2D quantum-well-based TIs considered in this work, the interplay of charge-carrier dynamics and the spinor character of extended bulk states could be a source of rich variety in spin-related properties also in $3 \mathrm{D}$, and the contributions of the conducting surfaces are currently not understood.

\section{ACKNOWLEDGMENTS}

E. M. H. acknowledges financial support from the German Science Foundation (DFG) via SFB 1170 "ToCoTronics," Grant No. HA 5893/4-1 within SPP 1666, and from the ENB Graduate School on Topological Insulators. E. M. H. and U. Z. started this work while visiting the Kavli Institute for Theoretical Physics at the University of Santa Barbara, where their work was supported in part by the National Science Foundation under Grant No. NSF PHY11-25915. The authors thank R. Winkler for illuminating discussions about possible forms of relevant spin operators and useful comments that have improved the manuscript.

\section{APPENDIX A: INTRINSIC CONTRIBUTION TO THE SPIN SUSCEPTIBILITY}

The intrinsic contributions to the diagonal entries of the spin susceptibility (the only ones that are nonzero for the BHZ model) are calculated by

$$
\bar{\chi}_{j j}^{(\mathrm{int})}(\gamma ; \mathbf{q})=-\sum_{\substack{s, s^{\prime} \\ \delta= \pm 1}} \int \frac{d^{2} k}{(2 \pi)^{2}} \frac{\mathcal{W}_{j j(\mathbf{k}, \mathbf{k}+\mathbf{q},+,-)}^{\left(s, s^{\prime}\right)}(\gamma) n_{\mathrm{F}}\left(E_{\mathbf{k}-}^{(s)}\right)}{E_{\mathbf{k}+}^{(s)}-E_{\mathbf{k}+\mathbf{q}-}^{\left(s^{\prime}\right)}+i \hbar \eta \delta},
$$



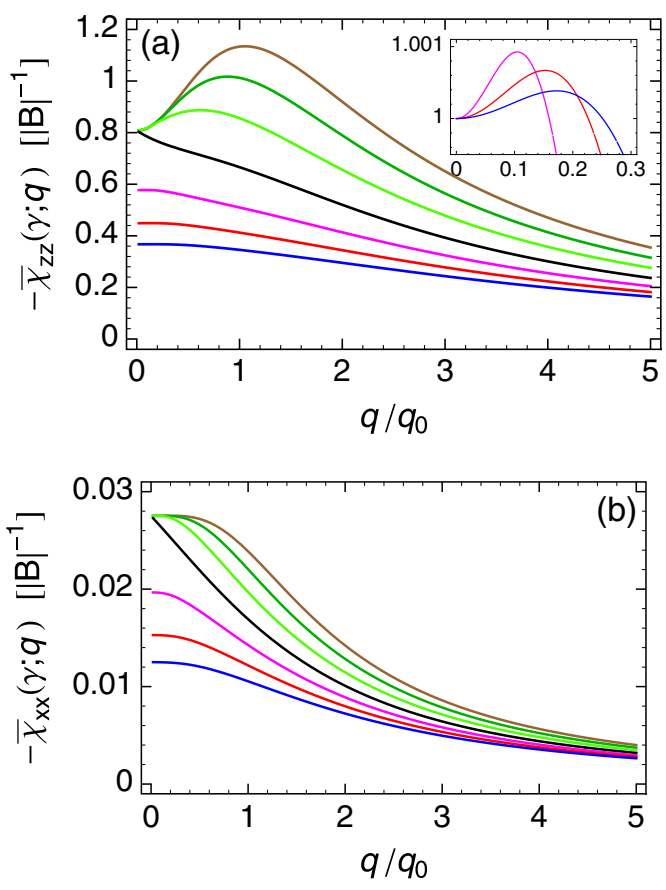

FIG. 6. Intrinsic spin susceptibilities (a) $\bar{\chi}_{z z}(\gamma ; q)$ and (b) $\bar{\chi}_{x x}(\gamma ; q)$ as a function of $q / q_{0}$ for various values of $\xi_{\mathrm{M}}=$ $(-0.3,-0.2,-0.1,0,0.1,0.2,0.3)$ (from top to bottom) with $\xi_{\mathrm{D}}=-0.7, \mathcal{C}_{\mathrm{LH}}=0.4$, and $\gamma=-2.22$. The inset in (a) displays the normalized spin susceptibility $\bar{\chi}_{z z}(\gamma ; q) / \bar{\chi}_{z z}(\gamma ; 0)$ in the small $q / q_{0}$ region for $\xi_{\mathrm{M}}=(0.1,0.2,0.3)$, which shows more clearly that $\bar{\chi}_{z z}(\gamma ; q)>\bar{\chi}_{z z}(\gamma ; 0)$ in the small- $q$ limit in the normal regime also.

where in the zero-temperature limit the valence band is fully occupied, i.e., $n_{\mathrm{F}}\left(E_{\mathbf{k}-}^{(s)}\right)=1$. Here and in the following, we consider the growth-direction-averaged case: $\bar{\chi}_{i j}(\gamma ; \mathbf{q})=$ $\int d z \int d z^{\prime} \chi_{i j}\left(\gamma ; \mathbf{q} ; z, z^{\prime}\right) \Rightarrow \mathcal{W}_{j j(\mathbf{k}, \mathbf{k}+\mathbf{q}, \alpha, \beta)}^{\left(s, s^{\prime}\right)}(\gamma)=\int d z d z^{\prime}$ $\mathcal{W}_{j j(\mathbf{k}, \mathbf{k}+\mathbf{q}, \alpha, \beta)}^{\left(s, s^{\prime}\right)}\left(\gamma ; z, z^{\prime}\right)$. In Fig. 6, we plot $\bar{\chi}_{z z}(\gamma ; q)$ $\left[\bar{\chi}_{x x}(\gamma ; q)\right]$ in panel (a) [(b)] as a function of $q$ for various values of $\xi_{\mathrm{M}}$. The inset in Fig. 6(a) illustrates that $\left.\left\{\left[\partial^{2} \bar{\chi}_{z z}^{\text {(int) }}(\gamma ; q)\right] /\left(\partial q^{2}\right)\right\}\right|_{q=0}<0$ for all of the values of $\xi_{\mathrm{M}}$ considered, which implies that the mean-field ferromagnetic order for out-of-plane aligned magnetic impurity spins will generally be destroyed by spin-wave (magnon) excitations [14].

\section{APPENDIX B: BLOEMBERGEN-ROWLAND BEHAVIOR OF THE LOCAL INTRINSIC SPIN SUSCEPTIBILITY}

Bloembergen and Rowland [34] found that the local RKKY interaction of gapped systems becomes short ranged, i.e., is exponentially suppressed by the band gap. The functional dependence of the local spin
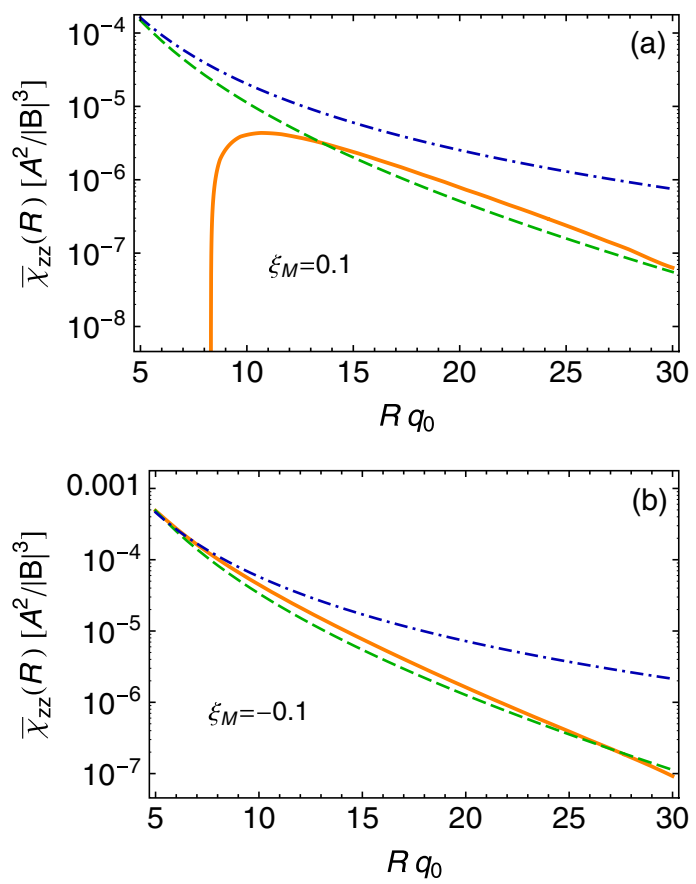

FIG. 7. Local intrinsic spin susceptibilities $\bar{\chi}_{z z}(R)$ (solid orange curve) as a function of $R q_{0}$ for (a) $\xi_{\mathrm{M}}=0.1$ and (b) $\xi_{\mathrm{M}}=-0.1$ with $\xi_{\mathrm{D}}=-0.7, \mathcal{C}_{\mathrm{LH}}=0.4$, and $\gamma=-2.22$. The dashed green curve shows the modeled behavior in Eq. (B1) with coefficients $c=1$ for (a) and $c=1.2$ for (b). For comparison, we also show the $R^{-3}$ decay for a gapless Dirac system represented by the dotdashed blue curve. The sharp drop of the orange curve in (a) is due to a sign change of $\bar{\chi}_{z z}(R)$ at about $R q_{0} \approx 8$.

susceptibility on the distance for the gapped Dirac system at hand can thus be modeled by

$$
\bar{\chi}_{j j}(R) \sim \frac{\mathrm{e}^{-c\left(R / \lambda_{\mathrm{C}}\right)}}{R^{3}},
$$

where $\lambda_{\mathrm{C}}^{-1} \equiv\left|\xi_{\mathrm{M}}\right| q_{0}$ is the inverse of the Compton wavelength of the system and $c \sim \mathcal{O}(1)$ is a numerical coefficient that can depend on the distance itself. To illustrate this behavior for the HgTe quantum-well system, we plot $\bar{\chi}_{z z}(R)$ in Fig. 7 as a function of $R q_{0}$ for $\xi_{\mathrm{M}}=0.1$ $\left[\xi_{\mathrm{M}}=-0.1\right]$ in panel (a) [(b)] together with both the line shape expected for 2D massless-Dirac particles and the Bloembergen-Rowland result.

\section{APPENDIX C: ELECTRON-DOPED CONTRIBUTION TO THE SPIN SUSCEPTIBILITY}

For the case where the Fermi energy is above the conduction energy band edge, i.e., $\mu>|M|$, the spin susceptibility receives contributions due to electron doping given by 

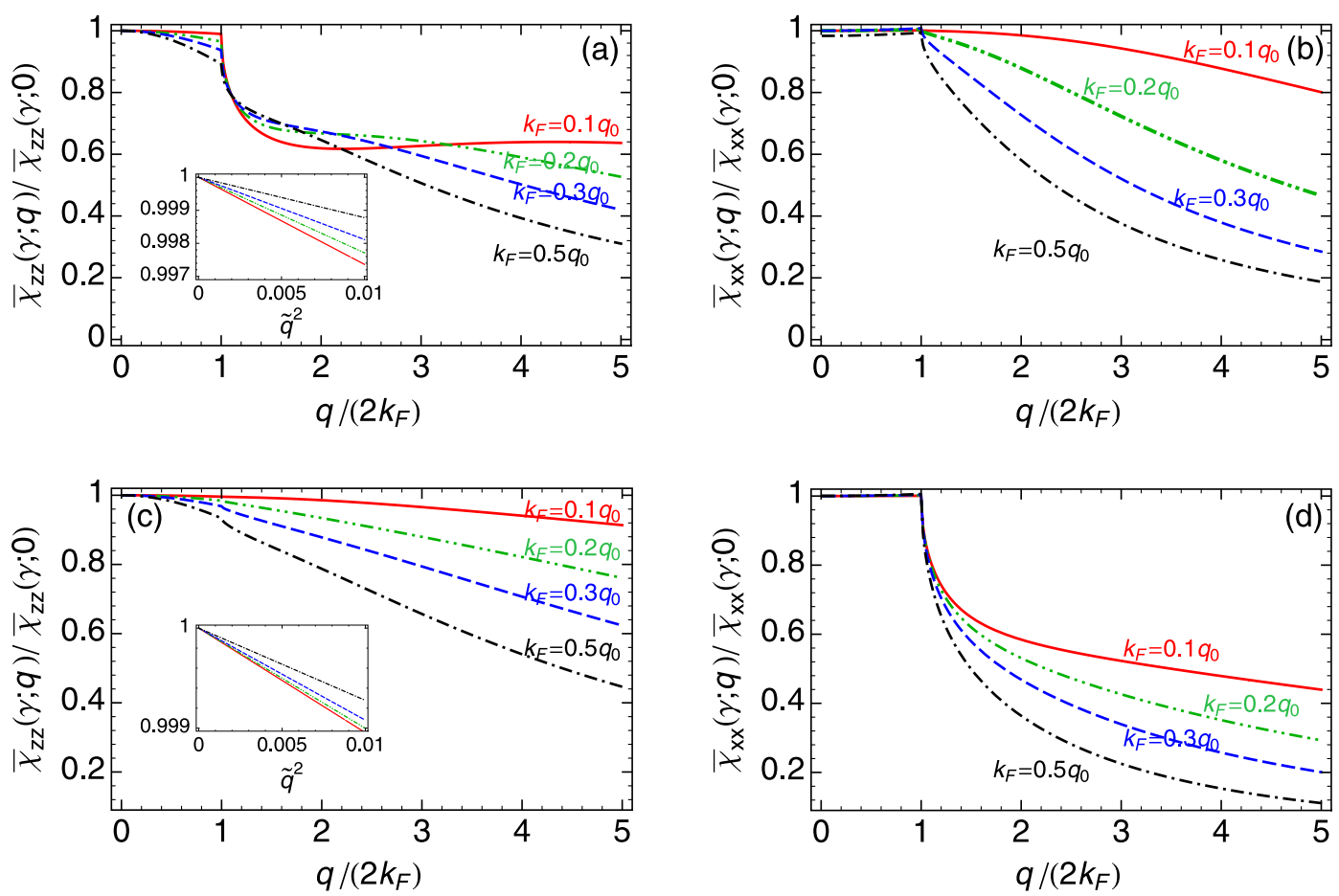

FIG. 8. Normalized spin susceptibilities $\bar{\chi}_{z z}(\gamma ; q)(\mathrm{a}, \mathrm{c})$ and $\bar{\chi}_{x x}(\gamma ; q)(\mathrm{b}, \mathrm{d})$ as a function of $q /\left(2 k_{\mathrm{F}}\right)$ for various levels of doping. (a,b) are the results for $\xi_{\mathrm{M}}=-0.2$ and (c,d) for $\xi_{\mathrm{M}}=0.2$, with $\xi_{\mathrm{D}}=-0.7, \mathcal{C}_{\mathrm{LH}}=0.4$, and $\gamma=-2.22$. The insets of (a,c) show the quadratic $\tilde{q}=q / q_{0}$ dependence close to $q=0$.

$$
\begin{aligned}
\bar{\chi}_{j j}^{(\mathrm{dop})}(\gamma ; \mathbf{q})= & \sum_{\substack{s, s^{\prime} \\
\delta= \pm 1}} \int \frac{d^{2} k}{(2 \pi)^{2}} n_{\mathrm{F}}\left(E_{\mathbf{k}+}^{(s)}\right)\left[\frac{\mathcal{W}_{j j(\mathbf{k}, \mathbf{k}+\mathbf{q},+,+)}^{\left(s, s^{\prime}\right)}(\gamma)}{E_{\mathbf{k}+}^{(s)}-E_{\mathbf{k}+\mathbf{q}+}^{\left(s^{\prime}\right)}+i \hbar \eta \delta}\right. \\
& \left.+\frac{\mathcal{W}_{j j(\mathbf{k}, \mathbf{k}+\mathbf{q},+,-)}^{\left(s, s^{\prime}\right)}(\gamma)}{E_{\mathbf{k}+}^{(s)}-E_{\mathbf{k}+\mathbf{q}-}^{\left(s^{\prime}\right)}+i \hbar \eta \delta}\right], \quad(\mathrm{C} 1)
\end{aligned}
$$

where for zero temperature $n_{\mathrm{F}}\left(E_{\mathbf{k}+}^{(s)}\right)=\Theta\left(k_{\mathrm{F}}-|\mathbf{k}|\right)$, with $k_{\mathrm{F}}$ being the Fermi wave vector associated with the conduction band. The complete spin susceptibility in the doped case is therefore given by

$$
\bar{\chi}_{j j}(\gamma ; \mathbf{q})=\bar{\chi}_{j j}^{(\mathrm{int})}(\gamma ; \mathbf{q})+\bar{\chi}_{j j}^{(\mathrm{dop})}(\gamma ; \mathbf{q}) .
$$

We show the line shape of $\bar{\chi}_{z z}(\gamma ; q)$ and $\bar{\chi}_{x x}(\gamma ; q)$ in Fig. 8 for $\xi_{\mathrm{M}}= \pm 0.2$ and various choices of doping. The insets of Figs. 8(a) and 8(c) show the small $q$ dependence of $\bar{\chi}_{z z}(\gamma ; q)$, indicating the stability of the out-of-plane Isingtype ferromagnetism with respect to magnon excitations.

\section{APPENDIX D: COMBINING BULK AND EDGE CONTRIBUTIONS TO THE SPIN SUSCEPTIBILITY}

In order to compare bulk and edge contributions to the spin susceptibility, we begin by considering the real-space spin-response function

$$
\chi_{i j}\left(\mathbf{r}, \mathbf{r}^{\prime}\right)=-\frac{i}{\hbar} \int_{0}^{\infty} d t \mathrm{e}^{-\eta t}\left\langle\left[S_{i}(\mathbf{r}, t), S_{j}\left(\mathbf{r}^{\prime}, 0\right)\right]\right\rangle,
$$

where $S_{i}(\mathbf{r})=\Psi^{\dagger}(\mathbf{r}) \hat{S}_{i} \Psi(\mathbf{r})$ are spin-density operators. In our system of interest, electrons are confined to move freely in $d<3$ dimensions, with system size $L$ in all of these free directions. The position vector is split up into a part $\mathbf{R}$ comprising the coordinate directions in which the motion is free and a part $\mathbf{Q}$ in whose coordinates motion is confined; $\mathbf{r}=(\mathbf{R}, \mathbf{0})$. The second-quantized electron operator in a real-space representation can be written as

$$
\Psi(\mathbf{r})=\sum_{\mathbf{k}, \alpha, s} \frac{\mathrm{e}^{i \mathbf{k} \cdot \mathbf{R}}}{\sqrt{L^{d}}} \psi_{\mathbf{k} \alpha}^{(s)}(\mathbf{0}) c_{\mathbf{k} \alpha}^{(s)}
$$

with normalized spinor bound-state wave functions $\psi_{\mathbf{k} \alpha}^{(s)}(\mathbf{0})$. A straightforward calculation yields

$$
\chi_{i j}\left(\mathbf{r}, \mathbf{r}^{\prime}\right)=\frac{1}{L^{d}} \sum_{\mathbf{q}} \mathrm{e}^{i \mathbf{q} \cdot\left(\mathbf{R}-\mathbf{R}^{\prime}\right)} \chi_{i j}^{(d \mathbf{D})}\left(\mathbf{q} ; \mathbf{o}, \mathbf{o}^{\prime}\right)
$$

with the q-dependent spin susceptibility of the $d$-dimensional $(d \mathrm{D})$ system given by 


$$
\begin{aligned}
\chi_{i j}^{(d \mathbf{D})}\left(\mathbf{q} ; \mathbf{0}, \mathbf{o}^{\prime}\right)= & \sum_{\alpha, \beta, s, s^{\prime}} \frac{1}{L^{d}} \sum_{\mathbf{k}} \mathcal{W}_{i j(\mathbf{k}, \mathbf{k}+\mathbf{q}, \alpha, \beta)}^{\left(s, s^{\prime}\right)}\left(\mathbf{0}, \mathbf{0}^{\prime}\right) \\
& \times \frac{n_{\mathrm{F}}\left(E_{\mathbf{k} \alpha}^{(s)}\right)-n_{\mathrm{F}}\left(E_{\mathbf{k}+\mathbf{q} \beta}^{\left(s^{\prime}\right)}\right)}{E_{\mathbf{k} \alpha}^{(s)}-E_{\mathbf{k}+\mathbf{q} \beta}^{\left(s^{\prime}\right)}+i \hbar \eta} .
\end{aligned}
$$

We are now interested in the homogeneous part of the spin response defined as

$$
\Upsilon_{i j}=\int d^{3} r \int d^{3} r^{\prime} \chi_{i j}\left(\mathbf{r}, \mathbf{r}^{\prime}\right) \equiv L^{d} \bar{\chi}_{i j}^{(d \mathrm{D})}(\mathbf{q}=0),
$$

where

$$
\bar{\chi}_{i j}^{(d \mathrm{D})}(\mathbf{q})=\int d^{3-d} \varrho \int d^{3-d} \varrho^{\prime} \chi_{i j}^{(d \mathrm{D})}\left(\mathbf{q} ; \mathbf{0}, \mathbf{0}^{\prime}\right)
$$

In the situation where both $2 \mathrm{D}$ bulk and $1 \mathrm{D}$ edge states are present, we therefore find

$$
\Upsilon_{i j}=L^{2}\left[\bar{\chi}_{i j}^{(2 \mathrm{D})}(\mathbf{q}=0)+\frac{1}{L} \bar{\chi}_{i j}^{(1 \mathrm{D})}(q=0)\right] .
$$

Thus, $L^{-2} \Upsilon$ is the well-defined quantity in the thermodynamic limit, and only those edge-related terms that scale with $L$ will contribute to it.

\section{APPENDIX E: SPIN SUSCEPTIBILITY OF EDGE STATES}

\section{Spin susceptibility of edge states: Omitting finite-size effects}

Following Ref. [5] (see also Ref. [44]), the dispersions for the edge states, using open boundary conditions for a confinement along the $x$ direction and assuming $D=0$, is given by

$$
E_{k}^{(s)}=s A k,
$$

where $k \equiv k_{y}$. The associated spinor wave functions are

$$
\eta^{(s)}(\mathbf{0})=\sum_{l=1}^{2} \varphi_{0, l}^{(s)}(x) \psi_{0 l}^{(s)}(z),
$$

where $\varphi_{0}^{(s)}(x)=C\left(\mathrm{e}^{\lambda_{1} x}-\mathrm{e}^{\lambda_{2} x}\right) \phi_{-s}$, with $\phi_{ \pm}^{T}=(1, \pm i)$ and $\lambda_{1,2}=-\frac{q_{0}}{2}\left(1 \pm \sqrt{1-4 \mid \xi_{\mathrm{M}}} \mid\right)$, since $M<0$. To simplify matters, we have assumed particle-hole symmetry $(D=0)$ and the system length $L \rightarrow \infty$; i.e., the gap in the dispersions of the edge states is negligible. Note that the spinors in Eq. (E2) are independent of the wave-vector component along the $y$ direction.
The spin susceptibility of the edge states is calculated by

$$
\begin{aligned}
\chi_{j j}^{(\text {edge })}\left(\gamma ; q ; \mathbf{0}, \mathbf{0}^{\prime}\right)= & \sum_{s, s^{\prime}} \int \frac{d k}{2 \pi} \mathcal{W}_{j j}^{\left(s, s^{\prime}\right)}\left(\gamma ; \mathbf{0}, \mathbf{o}^{\prime}\right) \\
& \times \frac{n_{\mathrm{F}}\left[E_{k}^{(s)}\right]-n_{\mathrm{F}}\left[E_{k+q}^{\left(s^{\prime}\right)}\right]}{E_{k}^{(s)}-E_{k+q}^{\left(s^{\prime}\right)}+i \hbar \eta},
\end{aligned}
$$

where

$$
\begin{aligned}
\mathcal{W}_{j j}^{\left(s, s^{\prime}\right)}\left(\gamma ; \mathbf{0}, \mathbf{\varrho}^{\prime}\right)= & {\left[\eta^{(s)}(\mathbf{Q})\right]^{\dagger} \cdot \hat{S}_{j}(\gamma) \cdot \eta^{\left(s^{\prime}\right)}(\mathbf{0}) } \\
& \times\left[\eta^{\left(s^{\prime}\right)}\left(\mathbf{\varrho}^{\prime}\right)\right]^{\dagger} \cdot \hat{S}_{j}(\gamma) \cdot \eta^{(s)}\left(\mathbf{\varrho}^{\prime}\right) .
\end{aligned}
$$

With an averaging over the coordinates along the confined directions, we have

$$
\begin{gathered}
\int d \mathbf{0} d \mathbf{\varrho}^{\prime} \mathcal{W}_{z z}^{\left(s, s^{\prime}\right)}\left(\gamma ; \mathbf{o}, \mathbf{o}^{\prime}\right)=\frac{\Xi_{z}^{2}(\gamma)}{16}\left(\begin{array}{ll}
1 & 0 \\
0 & 1
\end{array}\right), \\
\int d \mathbf{o} d \mathbf{\varrho}^{\prime} \mathcal{W}_{x x}^{\left(s, s^{\prime}\right)}\left(\gamma ; \mathbf{o}, \mathbf{o}^{\prime}\right)=\frac{\Xi_{x}^{2}(\gamma)}{16}\left(\begin{array}{ll}
0 & 1 \\
1 & 0
\end{array}\right),
\end{gathered}
$$

with $\Xi_{z}(\gamma)=1+3 \gamma+(\gamma-1) \mathcal{C}_{\mathrm{LH}} \quad$ and $\quad \Xi_{x}(\gamma)=1+$ $(2 \gamma-1) \mathcal{C}_{\mathrm{LH}} \equiv \mathcal{C}_{x}(\gamma) . \quad$ Thus, $\quad \chi_{z z}^{\text {(edge) }}(\gamma ; q) \propto \chi_{0}^{\text {(edge) }}(q)$, where $\chi_{0}^{\text {(edge) }}(q)$ is the Lindhard function associated with the edge states. Explicit calculation of Eq. (E3) yields

$$
\begin{array}{r}
\chi_{z z}^{\text {(edge) }}(\gamma ; q)=-\frac{\Xi_{z}^{2}(\gamma)}{16 \pi A} \equiv \frac{\Xi_{z}^{2}(\gamma)}{16} \chi_{0}^{(\text {edge })}(q), \\
\chi_{x x}^{(\text {edge })}(\gamma ; q)=\frac{\Xi_{x}^{2}(\gamma)}{32 \pi A} \ln \left(\frac{\left|q^{2}-4 k_{\mathrm{F}}^{2}\right|}{4 \Lambda^{2}-q^{2}}\right),
\end{array}
$$

where $\Lambda$ is a large-wave-vector cutoff. Because of the special energy dispersion, Eq. (E1), $\chi_{z z}^{(\text {edge })}(\gamma ; q)$ is a constant (independent of $k_{\mathrm{F}}$ and $q$ ). Clearly, for the hole-doped case $(\mu<0)$, the same result as in Eq. (E7) is obtained because of the assumed particle-hole symmetry.

\section{Spin susceptibility of edge states: Finite-size effects included}

Following Ref. [44], we now take into account the finite size of the system. As a result, edge states at the two sides that have the same spin can couple, which results in a gapped spectrum of their energy dispersions. We assume the system size $L$ to be large and use an approximation of the wave functions in Ref. [44]. Taking into account the various degrees of freedom of both system sides, we modify the approach of Ref. [5], where the solutions for the wave functions read 


$$
\varphi_{\nu}^{(s)}(x)=\frac{1}{\sqrt{C}}\left(\mathrm{e}^{\lambda_{1}[(L / 2)-s \nu x]}-\mathrm{e}^{\lambda_{2}[(L / 2)-s \nu x]}\right) \phi_{-s \nu}
$$

where $C$ is a normalization constant and $\nu= \pm$ denotes the right and left movers. For nonzero wave vector $k$, we consider the matrix

$$
\mathcal{H}_{\mathrm{RL}}=\left(\begin{array}{cc}
A k & \Delta \\
\Delta & -A k
\end{array}\right)
$$

in the basis of right and left movers, where $\Delta$ is the induced gap, which is a function of the system parameters [44]. The energy dispersions of Eq. (E9) are

$$
E_{k \tau}^{(s)}=\tau \sqrt{(A k)^{2}+\Delta^{2}}
$$

where $\tau= \pm$ labels the (spin-degenerate) conduction and valence bands, respectively. The associated eigenstates are

$$
a_{k \tau}^{(s)}=\left(\begin{array}{c}
\sqrt{\frac{E_{k \tau}^{(s)}+A k}{2 E_{k \tau}^{(s)}}} \\
\tau \sqrt{\frac{E_{k \tau}^{(s)}-A k}{2 E_{k \tau}^{(s)}}}
\end{array}\right) .
$$

With this information, the spinors in Eq. (E2) are modified to

$$
\eta_{k \tau}^{(s)}(\mathbf{0})=\sum_{l=1}^{2} \sum_{\nu} a_{k \tau, \nu}^{(s)} \varphi_{\nu, l}^{(s)}(x) \psi_{0 l}^{(s)}(z)
$$

Thus, for the present case, the spin susceptibility of the edge states is

$$
\begin{aligned}
\chi_{j j}^{(\text {edge })}\left(\gamma ; q ; \mathbf{0}, \mathbf{0}^{\prime}\right)= & \sum_{\substack{s, s^{\prime} \\
\tau, \tau^{\prime}}} \int \frac{d k}{2 \pi} \mathcal{W}_{j j\left(k, k+q, \tau, \tau^{\prime}\right)}^{\left(s, s^{\prime}\right)}\left(\gamma ; \mathbf{0}, \mathbf{0}^{\prime}\right) \\
& \times \frac{n_{\mathrm{F}}\left[E_{k \tau}^{(s)}\right]-n_{\mathrm{F}}\left[E_{k+q \tau^{\prime}}^{\left(s^{\prime}\right)}\right.}{E_{k \tau}^{(s)}-E_{k+q \tau^{\prime}}^{\left(s^{\prime}\right)}+i \hbar \eta},
\end{aligned}
$$

where

$$
\begin{aligned}
\mathcal{W}_{j j\left(k, k+q, \tau, \tau^{\prime}\right)}^{\left(s, s^{\prime}\right)}\left(\gamma ; \mathbf{Q}, \mathbf{Q}^{\prime}\right)= & {\left[\eta_{k \tau}^{(s)}(\mathbf{o})\right]^{\dagger} \cdot \hat{S}_{j}(\gamma) \cdot \eta_{k+q \tau^{\prime}}^{\left(s^{\prime}\right)}(\mathbf{o}) } \\
& \times\left[\eta_{k+q \tau^{\prime}}^{\left(s^{\prime}\right)}\left(\mathbf{Q}^{\prime}\right)\right]^{\dagger} \cdot \hat{S}_{j}(\gamma) \cdot \eta_{k \tau}^{(s)}\left(\mathbf{Q}^{\prime}\right) .
\end{aligned}
$$

Averaging over the coordinates along the confined directions, we obtain, for the overlap factor of the Lindhard function,

$$
\begin{aligned}
& \int d \mathbf{\varrho} d \mathbf{Q}^{\prime} \mathcal{W}_{0\left(k, k+q, \tau, \tau^{\prime}\right)}^{\left(s, s^{\prime}\right)}\left(\mathbf{\varrho}, \mathbf{\varrho}^{\prime}\right) \\
& \quad=\frac{\delta_{s s^{\prime}}}{4} \begin{cases}\left(a_{k} a_{k+q}+b_{k} b_{k+q}\right)^{2} & \left(\tau=\tau^{\prime}\right) \\
\left(a_{k} b_{k+q}-b_{k} a_{k+q}\right)^{2} & \left(\tau \neq \tau^{\prime}\right),\end{cases}
\end{aligned}
$$

where $\quad a_{k} \equiv \sqrt{1-\left[A k / \sqrt{(A k)^{2}+\Delta^{2}}\right]}, \quad b_{k} \equiv$ $\sqrt{1+\left[A k / \sqrt{(A k)^{2}+\Delta^{2}}\right]}$. The result for the overlap factors of the spin susceptibilities is

$$
\begin{aligned}
& \int d \mathbf{Q} d \mathbf{Q}^{\prime} \mathcal{W}_{z z\left(k, k+q, \tau, \tau^{\prime}\right)}^{\left(s, s^{\prime}\right)}\left(\gamma ; \mathbf{Q}, \mathbf{Q}^{\prime}\right) \\
& =\frac{\delta_{s s^{\prime}}}{64} \begin{cases}{\left[\left(a_{k} a_{k+q}+b_{k} b_{k+q}\right) \Xi_{z}(\gamma)\right.} & \left(\tau=\tau^{\prime}\right) \\
\left.+\tau\left(a_{k} b_{k+q}+b_{k} a_{k+q}\right) \mathcal{C}_{z}(\gamma) N L \Delta\right]^{2} & \\
{\left[\left(b_{k} b_{k+q}-a_{k} a_{k+q}\right) \mathcal{C}_{z}(\gamma) N L \Delta\right.} & \\
\left.+\tau\left(a_{k} b_{k+q}-b_{k} a_{k+q}\right) \Xi_{z}(\gamma)\right]^{2} & \end{cases}
\end{aligned}
$$

and

$$
\begin{aligned}
& \int d \mathbf{Q} d \mathbf{Q}^{\prime} \mathcal{W}_{x x\left(k, k+q, \tau, \tau^{\prime}\right)}^{\left(s, s^{\prime}\right)}\left(\gamma ; \mathbf{o}, \mathbf{Q}^{\prime}\right) \\
& =\frac{\Xi_{x}^{2}(\gamma)}{64}\left(1-\delta_{s s^{\prime}}\right)\left\{\begin{array}{ll}
{\left[a_{k} b_{k+q}+b_{k} a_{k+q}\right.} & \left(\tau=\tau^{\prime}\right) \\
\left.+\tau\left(a_{k} a_{k+q}+b_{k} b_{k+q}\right) N L \Delta\right]^{2} & \\
{\left[a_{k} a_{k+q}-b_{k} b_{k+q}\right.} & \\
\left.-\tau\left(a_{k} b_{k+q}-b_{k} a_{k+q}\right) N L \Delta\right]^{2} &
\end{array}\left(\tau \neq \tau^{\prime}\right) .\right.
\end{aligned}
$$

In obtaining Eqs. (E15)-(E17), we have used

$$
\int d x \prod_{\nu}\left(\mathrm{e}^{\lambda_{1}[(L / 2)-\nu x]}-\mathrm{e}^{\lambda_{2}[(L / 2)-\nu x]}\right) \approx L \mathrm{e}^{\lambda_{2} L},
$$

the functional $L$ dependence of $\Delta \approx F \exp \left(\lambda_{2} L\right)$ [44], where $F / E_{0}=4\left|\xi_{\mathrm{M}}\right| / \sqrt{1-4\left|\xi_{\mathrm{M}}\right|}$, and we have defined $N \equiv(F C)^{-1}$.

\section{a. Intrinsic contribution to the spin susceptibilities of the edge states in the limit $q \rightarrow 0$}

Calculating the intrinsic contribution to the spin susceptibilities of the edge states in the long-wavelength limit $(q \rightarrow 0)$, we obtain

$$
\begin{gathered}
\chi_{z z}^{(\text {int,e })}(\gamma ; 0)=(N L \Delta)^{2} \frac{g_{\mathrm{s}} \mathcal{C}_{z}^{2}(\gamma)}{16 \pi A}\left[1-\ln \left(\frac{2 \Lambda}{\tilde{\Delta}}\right)\right], \\
\chi_{x x}^{(\mathrm{int}, \mathrm{e})}(\gamma ; 0)=\frac{g_{\mathrm{s}} \Xi_{x}^{2}(\gamma)}{16 \pi A}\left[1-\ln \left(\frac{2 \Lambda}{\tilde{\Delta}}\right)\right],
\end{gathered}
$$


where $\tilde{\Delta} \equiv \Delta / A$. We note that the intrinsic contribution to the Lindhard function vanishes in the limit $q \rightarrow 0$, which can be inferred from Eq. (E15). To compare this result with the one of the intrinsic bulk contribution, we divide Eqs. (E19a) and (19b) by the length $L$ and let $L$ go to infinity (see Appendix D). Thus, we obtain

$$
\begin{gathered}
\lim _{L \rightarrow \infty} \frac{\chi_{z z}^{(\mathrm{int}, \mathrm{e})}(\gamma ; 0)}{L}=0 \\
\lim _{L \rightarrow \infty} \frac{\chi_{x x}^{(\mathrm{int}, \mathrm{e})}(\gamma ; 0)}{L}=-\frac{g_{\mathrm{s}} \Xi_{x}^{2}(\gamma)}{32 \pi|B|}\left(1-\sqrt{1-4\left|\xi_{\mathrm{M}}\right|}\right) .
\end{gathered}
$$

Therefore, the edge states give a contribution to the total susceptibility only for the in-plane component, and its sign equals that of the bulk contribution.

\section{b. Electron-doped contribution to the spin susceptibilities of the edge states in the limit $q \rightarrow 0$}

Next, we include the contributions due to doping to the spin susceptibilities. The interband contributions read (for $\left.A k_{\mathrm{F}} \gg \Delta\right)$

$$
\begin{gathered}
\chi_{z z}^{(\text {inter,e) }}(\gamma ; 0)=-(N L \Delta)^{2} \frac{g_{\mathrm{s}} \mathcal{C}_{z}^{2}(\gamma)}{16 \pi A}\left[1-\ln \left(\frac{2 k_{\mathrm{F}}}{\tilde{\Delta}}\right)\right], \\
\chi_{x x}^{(\text {inter,e) }}(\gamma ; 0)=-\frac{g_{\mathrm{s}} \Xi_{x}^{2}(\gamma)}{16 \pi A}\left[1-\ln \left(\frac{2 k_{\mathrm{F}}}{\tilde{\Delta}}\right)\right],
\end{gathered}
$$

while the intraband contributions are

$$
\begin{gathered}
\chi_{z z}^{(\text {intra,e) }}(\gamma ; 0)=-\frac{g_{\mathrm{s}} \Xi_{z}^{2}(\gamma)}{16 \pi A}, \\
\chi_{x x}^{(\text {intra,e) }}(\gamma ; 0)=-(N L \Delta)^{2} \frac{g_{\mathrm{s}} \Xi_{x}^{2}(\gamma)}{16 \pi A},
\end{gathered}
$$

which is consistent with the finding that $\chi_{z z}^{(\text {intra,e) }}(\gamma ; q)$ $\left[\chi_{x x}^{(\text {intra,e })}(\gamma ; q)\right]$ are important [unimportant] for $\Delta \rightarrow 0$, whereas it is the other way around for the interband contributions. Considering

$$
\begin{gathered}
\lim _{L \rightarrow \infty} \frac{\left[\chi_{z z}^{(\text {intra,e) }}(\gamma ; 0)+\chi_{z z}^{(\text {inter,e) }}(\gamma ; 0)\right]}{L}=0, \\
\lim _{L \rightarrow \infty} \frac{\left[\chi_{x x}^{(\text {intra,e) }}(\gamma ; 0)+\chi_{x x}^{(\text {inter }, \mathrm{e})}(\gamma ; 0)\right]}{L} \\
=\frac{g_{\mathrm{s}} \Xi_{x}^{2}(\gamma)}{32 \pi|B|}\left(1-\sqrt{1-4\left|\xi_{\mathrm{M}}\right|}\right),
\end{gathered}
$$

we find that this is the same contribution as the intrinsic contribution, Eq. (20b), which has, however, the opposite sign. Thus, the sum of doped and intrinsic contributions of the edge states vanishes in the large $L$ limit. As a consistency check, we verify for $q=0$ that the sum of Eqs. (E19), (E21), and (22) yields Eqs. (E7a) and (E7b) (multiplied by $g_{\mathrm{s}}$ ) in the limit $\Delta \rightarrow 0$.

\section{APPENDIX F: EFFECTS OF STRUCTURAL INVERSION ASYMMETRY}

Here, we demonstrate that the basic features of the intrinsic spin susceptibilities given in Eq. (1) are robust even when effects due to structural inversion asymmetry (SIA) are included. By taking into account the influence of a perpendicular electric field $\mathcal{E}_{z}$, it has been shown in Ref. [22] that the BHZ Hamiltonian is supplemented by entries that mix the spin-up and spin-down components of the BHZ basis states. The leading contribution due to SIA is linear in the wave vector and given by

$$
\mathcal{H}_{\mathrm{R}}=\left(\begin{array}{cccc}
0 & 0 & -i R_{0} k_{-} & 0 \\
0 & 0 & 0 & 0 \\
i R_{0} k_{+} & 0 & 0 & 0 \\
0 & 0 & 0 & 0
\end{array}\right)
$$

where $k_{ \pm}=k_{x} \pm i k_{y}$. The necessity to avoid dielectric breakdown provides an upper limit for the electric-field magnitude through the condition $\left|e \mathcal{E}_{z}\right| d<2|M|$. Defining the SIA-related dimensionless parameter $\xi_{\mathrm{R}} \equiv R_{0} / A$, this condition translates into $\left|\xi_{\mathrm{R}}\right|<16.1\left|\xi_{\mathrm{M}}\right| /(d[\mathrm{~nm}]) \approx 0.12$ for a typical heterostructure [22]. Thus, $\xi_{R}$ is generally a small parameter, and a perturbative treatment for SIA effects is appropriate. To lowest order in $\xi_{\mathrm{R}}$, the intrinsic spin susceptibility in the limit $q \rightarrow 0$ is found as

$$
\begin{aligned}
& \bar{\chi}_{x x}^{\text {(int) }}(\gamma ; \mathbf{q}=0) \\
& =-\frac{\mathcal{C}_{x}^{2}(\gamma)}{16 \pi|B|} \frac{1}{1+4 \xi_{\mathrm{M}} \Theta\left(\xi_{\mathrm{M}}\right)}\left(1+\xi_{\mathrm{R}}^{2} \frac{8 \xi_{\mathrm{M}} \Theta\left(\xi_{\mathrm{M}}\right)}{3\left[1+4 \xi_{\mathrm{M}} \Theta\left(\xi_{\mathrm{M}}\right)\right]^{2}}\right),
\end{aligned}
$$

$$
\begin{aligned}
& \bar{\chi}_{z z}^{\text {(int) }}(\gamma ; \mathbf{q}=0) \\
& =-\frac{\mathcal{C}_{z}^{2}(\gamma)}{16 \pi|B|} \frac{1}{1+4 \xi_{\mathrm{M}} \Theta\left(\xi_{\mathrm{M}}\right)} \\
& \quad \times\left(1+\xi_{\mathrm{R}}^{2} \frac{2\left\{9 \gamma^{2}+2 \xi_{\mathrm{M}} \Theta\left(\xi_{\mathrm{M}}\right)\left[\mathcal{C}_{z}^{2}(\gamma)+18 \gamma^{2}\right]\right\}}{3 \mathcal{C}_{z}^{2}(\gamma)\left[1+4 \xi_{\mathrm{M}} \Theta\left(\xi_{\mathrm{M}}\right)\right]^{2}}\right) .
\end{aligned}
$$

Thus, the lowest-order SIA corrections to $\bar{\chi}_{j j}^{\text {(int) }}(\gamma ; \mathbf{q}=0)$ are quadratic in the small parameter $\xi_{\mathrm{R}}$. This means that the result given in Eq. (F2) already represents an excellent approximation. Interestingly, $\bar{\chi}_{x x}^{\text {(int) }}(\gamma ; \mathbf{q}=0)$ turns out not to be modified by SIA contributions in the inverted regime. We find that this remains true even when higher-order 
corrections in $\xi_{\mathrm{R}}$ are considered. In contrast, $\bar{\chi}_{z z}^{\text {(int) }}(\gamma ; \mathbf{q}=0)$ in Eq. (F2b) has finite SIA corrections in the inverted regime given by $6 \gamma^{2} \xi_{\mathrm{R}}^{2} / \mathcal{C}_{z}^{2}(\gamma)$. In our case, where $\gamma=-2.22$, this amounts to a relative change that is about $1 \%$. Also in the normal regime, SIA contributions to $\bar{\chi}_{j j}^{\text {(int) }}(\gamma ; \mathbf{q}=0)$ are at most of relative magnitude $1 \%$. Thus, we conclude that the spin susceptibilities in Eq. (1) generally receive only very small corrections when SIA terms are included in the BHZ Hamiltonian.

[1] M. König, S. Wiedmann, C. Brüne, A. Roth, H. Buhmann, L. W. Molenkamp, X.-L. Qi, and S.-C. Zhang, Quantum Spin Hall Insulator State in HgTe Quantum Wells, Science 318, 766 (2007).

[2] A. Roth, C. Brüne, H. Buhmann, L. W. Molenkamp, J. Maciejko, X.-L. Qi, and S.-C. Zhang, Nonlocal Transport in the Quantum Spin Hall State, Science 325, 294 (2009).

[3] C. Brüne, A. Roth, H. Buhmann, E. M. Hankiewicz, L. W. Molenkamp, J. Maciejko, X.-L. Qi, and S.-C. Zhang, Spin Polarization of the Quantum Spin Hall Edge States, Nat. Phys. 8, 486 (2012).

[4] S. Hart, H. Ren, M. Kosowsky, G. Ben-Shach, P. Leubner, C. Brüne, H. Buhmann, L. W. Molenkamp, B. I. Halperin, and A. Yacoby, Controlled Finite Momentum Pairing and Spatially Varying Order Parameter in Proximitized HgTe Quantum Wells, arXiv:1509.02940.

[5] X.-L. Qi and S.-C. Zhang, Topological Insulators and Superconductors, Rev. Mod. Phys. 83, 1057 (2011).

[6] C. Liu, T. L. Hughes, X.-L. Qi, K. Wang, and S.-C. Zhang, Quantum Spin Hall Effect in Inverted Type-II Semiconductors, Phys. Rev. Lett. 100, 236601 (2008).

[7] I. Knez, R.-R. Du, and G. Sullivan, Evidence for Helical Edge Modes in Inverted InAs/GaSb Quantum Wells, Phys. Rev. Lett. 107, 136603 (2011).

[8] M. Z. Hasan and J. E. Moore, Three-Dimensional Topological Insulators, Annu. Rev. Condens. Matter Phys. 2, 55 (2011).

[9] A. M. Lunde and G. Platero, Hyperfine Interactions in TwoDimensional HgTe Topological Insulators, Phys. Rev. B 88, 115411 (2013).

[10] E. G. Novik, A. Pfeuffer-Jeschke, T. Jungwirth, V. Latussek, C. R. Becker, G. Landwehr, H. Buhmann, and L. W. Molenkamp, Band Structure of Semimagnetic $\mathrm{Hg}_{1-y} \mathrm{Mn}_{y}$ Te Quantum Wells, Phys. Rev. B 72, 035321 (2005).

[11] C.-X. Liu, X.-L. Qi, X. Dai, Z. Fang, and S.-C. Zhang, Quantum Anomalous Hall Effect in $\mathrm{Hg}_{1-y} \mathrm{Mn}_{y}$ Te Quantum Wells, Phys. Rev. Lett. 101, 146802 (2008).

[12] R. Yu, W. Zhang, H.-J. Zhang, S.-C. Zhang, X. Dai, and Z. Fang, Quantized Anomalous Hall Effect in Magnetic Topological Insulators, Science 329, 61 (2010).

[13] P. Simon and D. Loss, Nuclear Spin Ferromagnetic Phase Transition in an Interacting Two Dimensional Electron Gas, Phys. Rev. Lett. 98, 156401 (2007).

[14] P. Simon, B. Braunecker, and D. Loss, Magnetic Ordering of Nuclear Spins in an Interacting Two-Dimensional Electron Gas, Phys. Rev. B 77, 045108 (2008).
[15] T. Dietl, A Ten-Year Perspective on Dilute Magnetic Semiconductors and Oxides, Nat. Mater. 9, 965 (2010).

[16] G. Giuliani and G. Vignale, Quantum Theory of the Electron Liquid (Cambridge University Press, Cambridge, England, 2005).

[17] T. Kernreiter, M. Governale, and U. Zülicke, CarrierDensity-Controlled Anisotropic Spin Susceptibility of Two-Dimensional Hole Systems, Phys. Rev. Lett. 110, 026803 (2013).

[18] Q. Liu, C.-X. Liu, C. Xu, X.-L. Qi, and S.-C. Zhang, Magnetic Impurities on the Surface of a Topological Insulator, Phys. Rev. Lett. 102, 156603 (2009).

[19] J.-J. Zhu, D.-X. Yao, S.-C. Zhang, and K. Chang, Electrically Controllable Surface Magnetism on the Surface of Topological Insulators, Phys. Rev. Lett. 106, 097201 (2011).

[20] B. A. Bernevig, T. L. Hughes, and S.-C. Zhang, Quantum Spin Hall Effect and Topological Phase Transition in HgTe Quantum Wells, Science 314, 1757 (2006).

[21] J. Kossut and W. Dobrowolski, Properties of Diluted Magnetic Semiconductors, in Narrow Gap II-VI Compounds for Optoelectronic and Electromagnetic Applications, edited by P. Capper (Chapman \& Hall, London, 1997), pp. 401-429.

[22] D. G. Rothe, R. W. Reinthaler, C.-X. Liu, L. W. Molenkamp, S.-C. Zhang, and E. M. Hankiewicz, Fingerprint of Different Spin-Orbit Terms for Spin Transport in HgTe Quantum Wells, New J. Phys. 12, 065012 (2010).

[23] M. Mühlbauer, A. Budewitz, B. Büttner, G. Tkachov, E. M. Hankiewicz, C. Brüne, H. Buhmann, and L.W. Molenkamp, One-Dimensional Weak Antilocalization Due to the Berry Phase in HgTe Wires, Phys. Rev. Lett. 112, 146803 (2014).

[24] S. Juergens, P. Michetti, and B. Trauzettel, Plasmons Due to the Interplay of Dirac and Schrödinger Fermions, Phys. Rev. Lett. 112, 076804 (2014).

[25] S. Juergens, P. Michetti, and B. Trauzettel, Screening Properties and Plasmons of $\mathrm{Hg}(\mathrm{Cd}) \mathrm{Te}$ Quantum Wells, Phys. Rev. B 90, 115425 (2014).

[26] R. Winkler, Spin-Orbit Coupling Effects in TwoDimensional Electron and Hole Systems (Springer, Berlin, 2003).

[27] For example, when discussing Pauli paramagnetism, the response function for $\hat{S}_{i}(\gamma)$ with $\gamma=-2 \kappa / g_{*}$ is relevant, where $g_{*}$ and $\kappa$ are the respective $g$ factors for the $\Gamma_{6}$ and $\Gamma_{8}$ bands in the Kane-model band-structure description [26]. In contrast, the intrinsic real-spin polarization is associated with $\hat{S}_{i}(1 / 3)$. See, e.g., Eq. (6.65) in Ref. [26]. To discuss carrier-mediated magnetism, the susceptibility with $\gamma=\beta / \alpha$ in the notation of Ref. [21] needs to be considered. Finally, $\hat{S}_{i}(1)$ is the proper angular-momentum operator that generates spatial rotations.

[28] For instance, for a perpendicular magnetic field $\mathcal{B}=\mathcal{B}_{z} \hat{\mathbf{z}}$, the grand canonical potential is given by $J\left(T, \mathcal{B}_{z}, \mu\right)=$ $-\left(L^{2} / \beta\right) \sum_{\nu} \int\left[d^{2} k /(2 \pi)^{2}\right] \ln \left(1+\mathrm{e}^{-\beta\left[\varepsilon_{\nu}\left(k, \mathcal{B}_{z}\right)-\mu\right]}\right), \quad$ where $\beta^{-1}=k_{\mathrm{B}} T$ and $\varepsilon_{\nu}\left(k, \mathcal{B}_{z}\right)$ are the dispersions of the two spin-split bands $(\nu=1,2)$ obtained from the effective Hamiltonian. In the limit $T=0$ (and $\mu=0$ ), we obtain $J\left(\mathcal{B}_{z}\right)=\frac{L^{2}}{2 \pi} \sum_{\nu} \int_{0}^{\Lambda} d k k \varepsilon_{\nu}\left(k, \mathcal{B}_{z}\right)$. The net magnetization of the bands is given by $\mathcal{M}_{z}=\left(1 / L^{2}\right)\left[\partial J\left(\mathcal{B}_{z}\right) /\left(\partial \mathcal{B}_{z}\right)\right]$. Numerically, we find that $\left[\partial J\left(\mathcal{B}_{z}\right) /\left(\partial \mathcal{B}_{z}\right)\right] \propto \sum_{\nu} c_{\nu}(\Lambda)=0$ 
since the numerical coefficients of the two spin-split bands are related as $c_{1}(\Lambda)=-c_{2}(\Lambda)$.

[29] In other words, while the total magnetic susceptibility of the $\mathrm{HgTe}$ quantum-well system is trivial (i.e., vanishes) in the intrinsic limit, the effective-spin susceptibility is finite and exhibits interesting properties that affect observable physical phenomena, e.g., impurity-spin exchange and Zeeman splitting, as discussed in the following.

[30] The switching off of HH-LH mixing can be simulated by replacing $A \rightarrow \lambda A$ in Eq. (2) and letting $\lambda \rightarrow 0$ after integration.

[31] L. Zhou, J. Wiebe, S. Lounis, E. Vedmedenko, F. Meier, S. Blügel, P. H. Dederichs, and R. Wiesendanger, Strength and Directionality of Surface Ruderman-Kittel-Kasuya-Yosida Interaction Mapped on the Atomic Scale, Nat. Phys. 6, 187 (2010).

[32] A. A. Khajetoorians, J. Wiebe, B. Chilian, S. Lounis, S. Blügel, and R. Wiesendanger, Atom-by-Atom Engineering and Magnetometry of Tailored Nanomagnets, Nat. Phys. 8, 497 (2012).

[33] K. Yosida, Theory of Magnetism (Springer, Berlin, 1996).

[34] N. Bloembergen and T. J. Rowland, Nuclear Spin Exchange in Solids: $\mathrm{Tl}^{203}$ and $\mathrm{Tl}^{205}$ Magnetic Resonance in Thallium and Thallic Oxide, Phys. Rev. 97, 1679 (1955).

[35] T. Stauber, F. Guinea, and M. A. H. Vozmediano, Disorder and Interaction Effects in Two-Dimensional Graphene Sheets, Phys. Rev. B 71, 041406 (2005).

[36] C. Lewiner and G. Bastard, Indirect Exchange Interactions in Zero-Gap Semiconductors: Anisotropic Effects, Phys. Rev. B 22, 2132 (1980).

[37] V. I. Litvinov and V. K. Dugaev, Ferromagnetism in Magnetically Doped III-V Semiconductors, Phys. Rev. Lett. 86, 5593 (2001).

[38] R. A. Żak, D. L. Maslov, and D. Loss, Ferromagnetic Order of Nuclear Spins Coupled to Conduction Electrons: A
Combined Effect of Electron-Electron and Spin-Orbit Interactions, Phys. Rev. B 85, 115424 (2012).

[39] E. Tutuc, S. Melinte, and M. Shayegan, Spin Polarization and $g$ Factor of a Dilute GaAs Two-Dimensional Electron System, Phys. Rev. Lett. 88, 036805 (2002).

[40] J. Zhu, H. L. Stormer, L. N. Pfeiffer, K. W. Baldwin, and K. W. West, Spin Susceptibility of an Ultra-Low-Density Two-Dimensional Electron System, Phys. Rev. Lett. 90, 056805 (2003).

[41] A. A. Shashkin, S. Anissimova, M. R. Sakr, S. V. Kravchenko, V. T. Dolgopolov, and T. M. Klapwijk, Pauli Spin Susceptibility of a Strongly Correlated TwoDimensional Electron Liquid, Phys. Rev. Lett. 96, 036403 (2006).

[42] M. König, H. Buhmann, L. W. Molenkamp, T. Hughes, C.-X. Liu, X.-L. Qi, and S.-C. Zhang, The Quantum Spin Hall Effect: Theory and Experiment, J. Phys. Soc. Jpn. 77, 031007 (2008).

[43] R. Winkler, D. Culcer, S. J. Papadakis, B. Habib, and M. Shayegan, Spin Orientation of Holes in Quantum Wells, Semicond. Sci. Technol. 23, 114017 (2008).

[44] B. Zhou, H.-Z. Lu, R.-L. Chu, S.-Q. Shen, and Q. Niu, Finite Size Effects on Helical Edge States in a Quantum Spin-Hall System, Phys. Rev. Lett. 101, 246807 (2008).

[45] H. Hatami, T. Kernreiter, and U. Zülicke, Spin Susceptibility of Two-Dimensional Transition-Metal Dichalcogenides, Phys. Rev. B 90, 045412 (2014).

[46] S. Hart, H. Ren, T. Wagner, P. Leubner, M. Mühlbauer, C. Brüne, H. Buhmann, L. W. Molenkamp, and A. Yacoby, Induced Superconductivity in the Quantum Spin Hall Edge, Nat. Phys. 10, 638 (2014).

[47] V. S. Pribiag, A. J. A. Beukman, F. Qu, M. C. Cassidy, C. Charpentier, W. Wegscheider, and L. P. Kouwenhoven, Edge-Mode Superconductivity in a Two-Dimensional Topological Insulator, Nat. Nanotechnol. 10, 593 (2015). 